Draft Version May 19, 2020

Preprint typeset using $\mathrm{LAT}_{\mathrm{E}} \mathrm{X}$ style emulateapj v. 12/16/11

\title{
A COMPARISON OF X-RAY PHOTON INDICES AMONG THE NARROW AND BROAD-LINE SEYFERT 1 GALAXIES
}

\author{
Vineet Ojha ${ }^{1}$, Hum Chand ${ }^{1,2}$, Gulab Chand Dewangan ${ }^{3}$, Suvendu Rakshit $^{4}$ \\ ${ }^{1}$ Aryabhatta Research Institute of Observational Sciences (ARIES), Manora Peak, Nainital - 263001, India \\ ${ }^{2}$ Department of Physics and Astronomical Sciences, Central University of Himachal Pradesh (CUHP), Dharamshala-176215, India \\ ${ }^{3}$ Inter-University Centre for Astronomy and Astrophysics (IUCAA), Pune - 411007, India \\ ${ }^{4}$ Finnish Centre for Astronomy with ESO (FINCA), University of Turku, Quantum, Vesilinnantie 5, 20014 University of Turku, Finland \\ vineetojhabhu@gmail.com
}

Draft version May 19, 2020

\section{ABSTRACT}

We present a detailed comparative systematic study using a sample of 221 Narrow-line Seyfert 1 (NLSy1) galaxies in comparison to a redshift matched sample of 154 Broad-line Seyfert 1 (BLSy1) galaxies based on their observations using ROSAT and/or XMM-Newton telescopes in soft X-ray band (0.1-2.0 keV). A homogeneous analysis is carried out to estimate their soft X-ray photon indices $\left(\Gamma_{X}^{s}\right)$ and its correlations with other parameters of nuclear activities such as Eddington ratios $\left(\mathrm{R}_{\mathrm{Edd}}\right)$, bolometric luminosities $\left(\mathrm{L}_{\mathrm{bol}}\right)$, black hole masses $\left(\mathrm{M}_{\mathrm{BH}}\right)$ and the widths of the broad component of $\mathrm{H} \beta$ lines $(\operatorname{FWHM}(\mathrm{H} \beta))$. In our analysis, we found clear evidence of the difference in the $\Gamma_{X}^{s}$ and $\mathrm{R}_{\mathrm{Edd}}$ distributions among NLSy1 and BLSy1 galaxies, with steeper $\Gamma_{X}^{s}$ and higher $R_{\text {Edd }}$ for the former. Such a difference also exists in the spectral indices distribution in hard X-ray $\left(\Gamma_{X}^{h}\right)$, based on the analysis of 53 NLSy1 and 46 BLSy1 galaxies in the 2-10 keV energy band. The difference in $R_{E d d}$ distributions does exist even after applying the average correction for the difference in the inclination angle of NLSy1 and BLSy1 galaxies. We also estimated $R_{\text {Edd }}$, based on SED fitting of 34 NLSy1 and 30 BLSy1 galaxies over the $0.3-10 \mathrm{keV}$ energy band and found that results are still consistent with $\mathrm{R}_{\mathrm{Edd}}$ estimates based on the optical bolometric luminosity. Our analysis suggests that the higher $\mathrm{R}_{\text {Edd }}$ in NLSy1 is responsible for its steeper X-ray spectral slope compared to the BLSy1, consistent with the disc-corona model as proposed for the luminous AGNs.

Subject headings: surveys-galaxies: active-galaxies: accretion discs-galaxies: Seyfert-gamma-rays: galaxies active galaxies: nuclei-X-rays: galaxies.

\section{INTRODUCTION}

Narrow-line Seyfert 1 galaxies (NLSy1s) are a peculiar class of lower-luminosity Active Galactic Nuclei (AGNs), as defined by the width of the broad component of $\mathrm{H} \beta \quad(\mathrm{FWHM}(\mathrm{H} \beta)) \lesssim 2000 \mathrm{~km} \mathrm{~s}^{-1}$, flux ratio of $\left[\mathrm{O}_{I I I}\right]_{\lambda 5007} / H \beta \lesssim 3$ and strong permitted optical/UV Fe II emission lines (Shuder \& Osterbrock 1981; Osterbrock \& Pogge 1985; Boroson \& Green 1992; Grupe et al. 1999). They show steep soft X-ray spectra and rapid X-ray flux variability (Boller et al. 1996; Wang et al. 1996; Grupe et al. 1998; Leighly 1999; Komossa \& Meerschweinchen 2000; Miller et al. 2000; Klimek et al. 2004). Observations suggest that NLSy1s tend to have smaller black hole masses $\left(\mathrm{M}_{\mathrm{BH}}\right)$ and higher Eddington ratios (defined as the ratio of bolometric-to-Eddington luminosity as $\left.R_{\mathrm{E} d d} \equiv L_{\mathrm{bol}} / L_{\mathrm{E} d d}\right)$ compared to the broad line AGNs (Boroson \& Green 1992; Pounds et al. 1995; Sulentic et al. 2000; Boroson 2002; Collin \& Kawaguchi 2004). On the other hand, Gayathri et al. (2019) reported a similarity of $\mathrm{R}_{\mathrm{E} d d}$ and $\mathrm{M}_{\mathrm{B} H}$ among NLSy1s and Broad-line Seyfert 1 galaxies (BLSy1s) based on the accretion disc (AD) modeling of their optical spectra. Comparatively little is known about the intrinsic emission mechanisms of NLSy1s which are responsible for their aforementioned properties. However, since the launch of the many space telescopes such as ROent- gen SATellite (ROSAT), Chandra, X-ray Multi-Mirror Mission-Newton (XMM-Newton), and Fermi Large Area Telescope (LAT), many NLSy1s have been detected in the high energy bands such as X-rays and $\gamma$-rays. These high energy emissions are thought to be one of the most direct forms of nuclear activity which do play a crucial role in understanding the accretion process in the different types of AGNs.

For instance, a remarkable correlation has been found by Boller et al. (1996) \& Wang et al. (1996) between the soft X-ray photon indices and the widths of the broad component of $\mathrm{H} \beta$ lines $(\mathrm{FWHM}(\mathrm{H} \beta))$ in the NLSy1s. This is interpreted with the variation of accretion rate in different objects (Wandel et al. 1985; Pounds et al. 1994). To test this hypothesis, Lu \& Yu (1999) have compiled a sample of Seyfert 1 galaxies, QSOs, and found that the soft X-ray photon indices strongly correlate with the accretion rates. Additionally, Laor et al. (1997) have found a correlation between the soft X-ray $(0.2-2.0 \mathrm{keV})$ slope and the FWHM of the $\mathrm{H} \beta$ emission line in a sample of 23 low-redshift quasars suggesting that the physical parameter driving the correlation is the Eddington ratio. Many past X-ray studies of the Low luminosity AGNs (LLAGNs, comprising low-ionization nuclear emission-line regions and local Seyfert galaxies), have been carried out to explore any correlation among X-ray photon indices with other parameters of nuclear activities (e.g., see González-Martín et al. 2006; Panessa et al. 
2006; Gu \& Cao 2009). For instance, Gu \& Cao (2009) find a significant anticorrelation among the hard X-ray photon indices and the Eddington ratios using a sample of 55 LLAGNs, whose X-ray photon indices are collected from the literature having Chandra or XMMNewton observations. This anticorrelation resembles the spectra produced from advection dominated accretion flows (ADAFs) model for the X-ray binaries (XRBs) in the low state (e.g., see Esin et al. 1997). However it is found in contrast with the positive correlation reported by Risaliti et al. (2009) for the luminous AGNs. Their analysis led to an important suggestion that the spectra of LLAGNs might be produced by Comptonization process in ADAFs, which is similar to that of XRBs but is different from that in luminous AGNs. As a result, such analysis has important implications for the physical link between the accretion efficiency in the (cold) accretion disk of AGNs and the physical status of the (hot) corona.

For the X-ray detected NLSy1 galaxies, their Xray $/ \gamma$-ray emissions can be either from the jets whose existence is inferred based on their high variability in short time scales (Paliya et al. 2014; Kshama et al. 2017; Ojha et al. 2019), or it could be based on the ADAFs mechanism as suggested by $\mathrm{Gu}$ \& Cao (2009) for LLAGNs. Another possibility could be the accretionflow/hot-corona system of radiatively efficient accretion, as suggested by Maoz (2007) where thin accretion disc may persist at lower accretion rates. Additionally, it could also be from the widely accepted disk-corona model. In this model, UV soft photons from the accretion disc are comptonized and up-scattered (inverse Comptonization) into the X-ray bands by a hot corona, existing above the accretion disc (Haardt \& Maraschi 1991, 1993). To get an insight into the emissions from the central engine of NLSy1s, one possibility is to compare the distribution of its key parameters such as $\mathrm{R}_{\mathrm{E} d d}$, $\mathrm{M}_{B H}$, and X-ray spectral slopes, etc, with the control sample of BLSy1s matching in luminosity-redshift (L-z) plane. Any observational constraints based on such comparisons, can be very useful to probe the above possible mechanisms. For instance, if the $\mathrm{R}_{\mathrm{E} d d}$ of NLSy1s in comparison to BLSy1s are statistically higher then one would expect an increase in the disk temperature, hence the production of more X-ray radiations, and at the same time, it can also increase the Compton cooling of the corona (Haardt \& Maraschi 1991, 1993; Zdziarski et al. 2000; Kawaguchi et al. 2001). This can further lead to observable steepening of the X-ray power-law more in NLSy1s than BLSy1s. Therefore, for such an insight especially about the X-ray emissions mechanism, X-ray spectral slope (both in the soft and hard X-ray energy bands) of a statistical large sample of NLSy1s along with its control sample of BLSy1s (matching in their L-Z plane) is very useful. This can also help to parametrize the cooling and heating mechanism of the X-ray corona, along with the underlying electrons' energy distribution.

However, the main hindrance till now in the aforementioned investigations was the lack of reasonable statistical homogeneous sample (see Brandt et al. 1997) added by a homogeneous analysis in the soft $(0.1-2.0 \mathrm{keV})$ and the hard $(2-10 \mathrm{keV})$ X-ray bands for the NLSy1 and BLSy1 galaxies, preferably matching in L-z plane. This was due to a relatively small available sample size of a total of 2000 optically detected NLSy1s given by Zhou et al. (2006) based on Sloan Digital Sky Survey (SDSS) Data Release 3 (SDSS, DR-3 Schneider et al. 2005). In contrast, based on a 10 fold increase in the number of AGNs in SDSS spectroscopic data release 12 (SDSS DR-12, Alam et al. 2015) than the DR-3. Rakshit et al. (2017) have recently enlarged the sample of NLSy1s to a total of 11,101 objects which is about 5 times larger than the number of previously known NLSy1 galaxies based on Zhou et al. (2006) catalog. This enlarged sample can be used to carry out a systematic and homogeneous analysis of a statistical sample of NLSyls (both in optical and X-ray). This can further also be used to compare its key parameters of nuclear activities, such as $\mathrm{R}_{\mathrm{E} d d}$, $\mathrm{M}_{B H}$ and $\mathrm{X}$-ray spectral slopes with a control sample of BLSy1s (preferably match in their L-z plane). This analysis is also favorable to investigate whether the steepening reported in the spectral slopes of NLSy1s (albeit deduced with small sample size, e.g., see Brandt et al. 1997) as compared to BLSy1s exist only in soft X-ray band or also extend to the hard X-ray band which is less prone to the soft X-ray excess (Boller et al. 1996; Brandt et al. 1997; Vaughan et al. 1999; Boller et al. 2002; Czerny et al. 2003; Vignali et al. 2004).

Here, we have worked towards the aforementioned goals. For this, we have selected a sample of 221 NLSy1s by cross-correlating 11,101 NLSy1s with that of the second ROSAT all-sky survey (2RXS) source catalog of Boller et al. (2016) and based on any source observation in XMM-Newton, available on HEASARC public data $\operatorname{archive}^{1}$ (e.g., Sect. 2). The corresponding control sample of 154 BLSy1s in the X-ray band, moderately matching in the redshift with that of our NLSy1s (e.g. Sect. 2) sample is used to carry out the comparative study of these two subclasses.

For the homogeneous X-ray analysis of the NLSy1 and BLSy1 galaxies, similar models and homogeneous methods are adopted for estimating their X-ray spectral slopes both in the soft and hard X-ray bands. In the same way, a homogeneous method is also applied to estimate the black-hole masses for all the members of our samples by careful modeling of the $\mathrm{H} \beta$ lines using their SDSS optical spectra. This is used to investigate any statistical relationships among the X-ray photon indices of NLSy1s and BLSy1s with their other key parameters of nuclear activities such as $\mathrm{FWHM}(\mathrm{H} \beta), M_{\mathrm{B} H}$, bolometric luminosities $\left(\mathrm{L}_{\mathrm{bol}}\right)$ and $\mathrm{R}_{\mathrm{E} d d}$. This allows us to understand the $\mathrm{X}$ ray emission mechanisms of NLSy1s as compared to the BLSy1s, along with the comparison of their properties with other luminous AGNs.

The paper is structured as follows. Sect. 2 describes the data sample and selection criteria. Sect. 3 describes observations and data reduction along with our analysis for X-ray data. Sect. 4 gives details of our spectral analysis. In Sect. 5, we focus on our results while discussion and conclusion are given in Sect. 6. Finally, we summarize our work in Sect. 7. Throughout, we have used a cosmology with $\Omega_{m}=0.286, \Omega_{\lambda}=0.714$, and $H_{o}=69.6 \mathrm{kms}^{-1} \mathrm{Mpc}^{-1}$ (Bennett et al. 2014).

\section{SAMPLE SELECTION}

\footnotetext{
1 https://heasarc.gsfc.nasa.gov/db-perl/W3Browse/w3browse.pl
} 

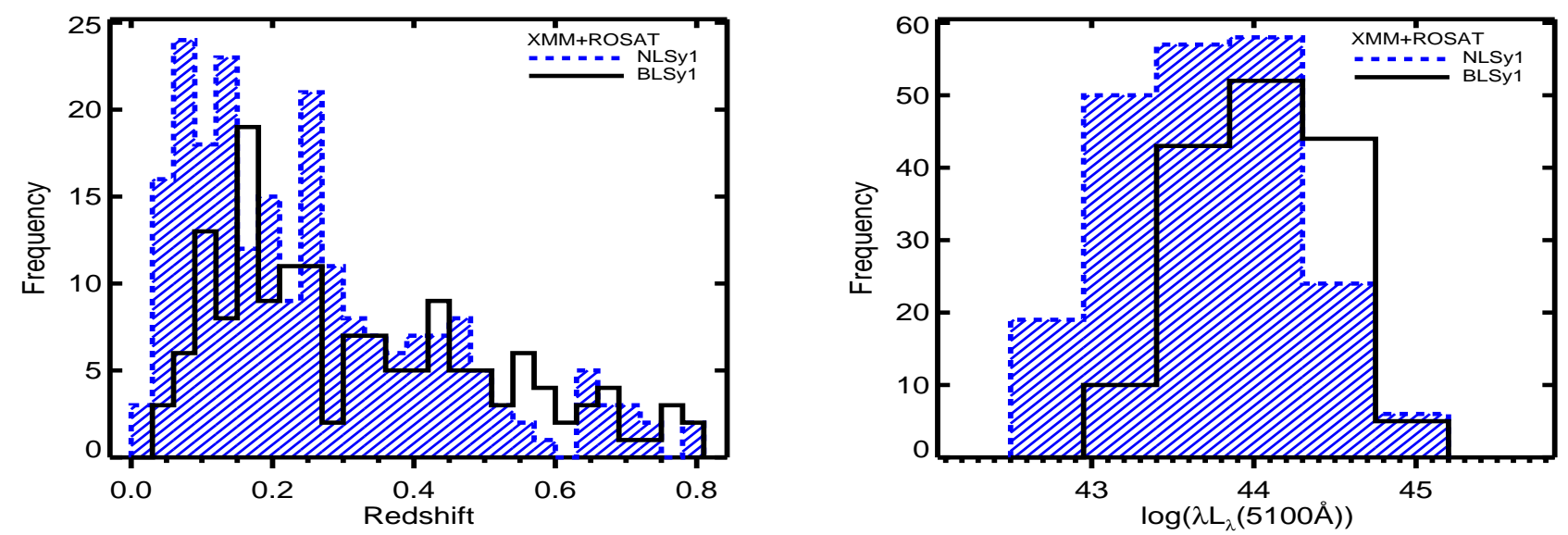

FIG. 1.- Distribution of emission redshifts (left) and $\lambda L_{\lambda}(5100 \AA)$ (right) for our XMM-Newton and ROSAT detected combined samples of 221 NLSy1s (blue, filled) and 154 BLSy1s (black, solid line).

For constructing our sample of NLSy1 galaxies, we used a recent catalog of NLSy1s given by Rakshit et al. (2017) in which they have compiled 11,101 NLSy1s using the SDSS DR-12 database. To make a sample of X-ray detected NLSy1s, we have cross-correlated these 11,101 NLSy1s with the 2RXS source catalog. This crosscorrelation resulted in the 1873 matches in the 2RXS catalog within a position offset (in sources RA and DEC) of 30 arcseconds. Similarly, we also searched for any XMMNewton telescope based observations for the above sample of 11,101 NLSy1s by using the HEASARC public data archive. This resulted in a sample of 697 XMM-Newton observed NLSy1s such that each of the NLSy1 falls within the $27.5 \mathrm{arcmin}^{2}$ offset from the pointing center of the parent XMM-Newton observation. Here, for any source with multiple observation IDs, the repetition is avoided by retaining only the observation with the largest observing time. We also noticed that like XMM-Newton the above energy range is also covered by Chandra telescope but due to its much smaller effective area (e.g., 600 $\left.\mathrm{cm}^{2}\right)$ as compared to XMM-Newton $\left(\sim 1227 \mathrm{~cm}^{2}\right)$, a typical increase in sample size due to the observed sources by the Chandra telescope is found to be nominal (around $\sim 10 \%$ ). Therefore, we have limited our analysis only to $X M M$-Newton's covered sources and the ROSAT 2RXS catalog's matched sources only.

Observations in the ROSAT were carried out using two detectors, viz., position-sensitive proportional counter (PSPC) and High-Resolution Imager (HRI). Furthermore, we noted that the HRI is essentially an imager with very little spectral response. Therefore, we limited our ROSAT sample, only to those sources which were observed with the PSPC instrument. This filter reduces our sample of ROSAT detected sources (hereafter ROSAT) from 1873 to 530 sources.

The 0.1-2.0 keV ROSAT spectrum of each source was extracted using standard XSELECT tasks of the HEASOFT software (version 6.25) with the appropriate circular region around the source to enhance source signal and reduce the background noise. This is found to differ for different sources depending on the number of pixels containing the maximum flux of the source (e.g. Sect. 3). The impact of this choice of the aperture by the eye on the signal-to-noise $(\mathrm{S} / \mathrm{N})$, as well as on our analysis (since for both same aperture is used) is found to be negligible in our sample. However, a very nominal enhancement in $\mathrm{S} / \mathrm{N}$ is found due to relatively less background noise in comparison to a fixed (50 arcseconds) aperture encircling about $90 \%$ energy fraction.

To exclude sources without high quality data, we have put a minimum $\mathrm{S} / \mathrm{N}$ criterian of 10 on our sample. For computing the $\mathrm{S} / \mathrm{N}$, we have estimated $\left[N_{s r c}-\right.$ $\left.N_{b k g}\right] / \sqrt{\left(N_{s r c}+N_{b k g}\right)}$, where $N_{s r c}$ and $N_{b k g}$ refer to total count contributed by aperture around the source and background region. Here the background region is chosen in close proximity to the source with aperture size fixed to its value as used for extracting the source count. This aperture size could either be fixed so that it encircled about $90 \%$ energy fraction or can also be optimized to enhance the $\mathrm{S} / \mathrm{N}$, as with an increase in aperture size background noise also increases. We have opted to use the latter, though the increase in $\mathrm{S} / \mathrm{N}$ using it is found to be nominal in comparison to the former method (e.g. with 50 arcsec fixed aperture to encircle $90 \%$ energy). We also note that the $\mathrm{S} / \mathrm{N}$ computed by the $\left[N_{s r c}-N_{b k g}\right] / \sqrt{\left(N_{s r c}+N_{b k g}\right)}$ method is consistent with that using [count rate]/[error on count rate] as return by XSPEC (version 6.25) for the source 'grp' file. For our ROSAT sources, we used 0.1-2.0 $\mathrm{keV}$ energy range while computing the $\mathrm{S} / \mathrm{N}$ using the above [count rate]/[error on count rate] method. The $\mathrm{S} / \mathrm{N} \geq 10$ criterion was satisfied by 83 out of 530 (henceforth also referred to as 83/530) ROSAT/PSPC (0.1-2.0 $\mathrm{keV})$ detected sources. For the 697 XMM-Newton detected (hereafter $X M M$ ) NLSy1s, among its three European Photon Imaging Camera (EPIC) detectors, we have limited our analysis only to $\mathrm{PN}$ detector due to its larger effective area (about $1227 \mathrm{~cm}^{2}$ at $1 \mathrm{keV}$ ). Each source spectrum was extracted over the appropriate circular aperture, selected by the eye around the source, in the same way as we had done for ROSAT (e.g. Sect. 3). The $\mathrm{S} / \mathrm{N} \geq 10$ criterion was satisfied by $148 / 697 X M M / P N$ sources in (0.3-2.0 keV) energy band. Further, we noticed that 8 sources are common between the samples of 83 ROSAT selected NLSy1s and the 148 XMM NLSy1s. For these 8 sources, we have used only XMM-Newton 

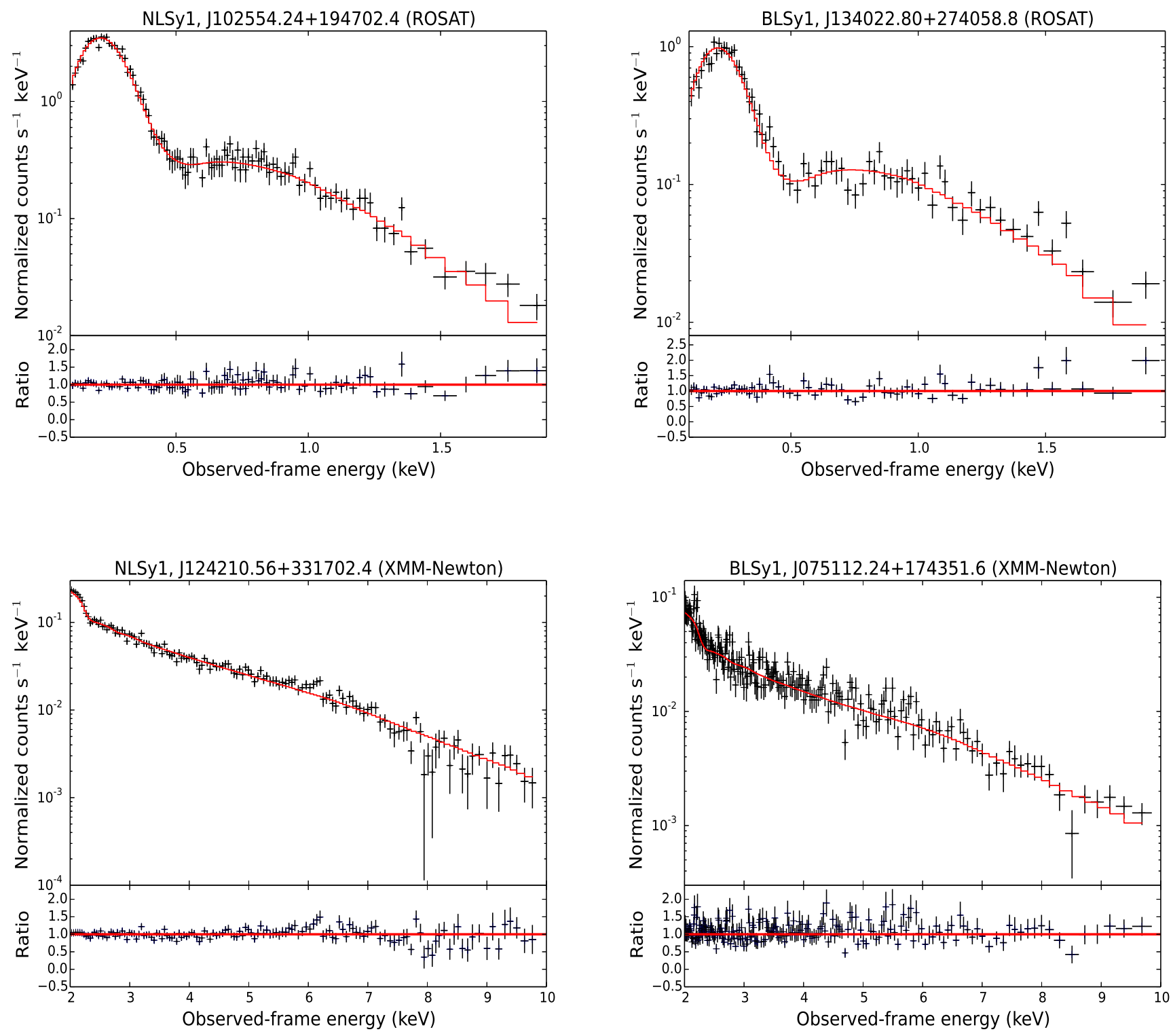

Fig. 2.- Top: Representative ROSAT soft (0.1-2.0 keV)/PSPC data and best fit folded models for the NLSy1 galaxy, viz., J102554.24+194702.4 (top left panel) and BLSy1 galaxy, viz., J134022.80+274058.8 (top right panel). In each case, our fit was carried out using power-law and double neutral absorption models. Bottom: The same as the top panels but for the XMM-Newton hard (2-10 keV)/PN data of the NLSy1 galaxy, viz., J124210.56+331702.4 (bottom left panel) and BLSy1 galaxy, viz., J075112.24+174351.6 (bottom right panel).

telescope observations due to its better spectral resolution and effective area (about $1227 \mathrm{~cm}^{2}$ at $1 \mathrm{keV}$ ) as compared to the ROSAT telescope (about $240 \mathrm{~cm}^{2}$ at $1 \mathrm{keV}$ ). This led to our final sample of 223 sources with 75 from the ROSAT and 148 from the XMM-Newton telescopes for their further X-ray spectral fitting.

To make a sample of BLSy1 galaxies matching in Lz plane with our above sample of NLSy1 galaxies, so as to carry out their comparative study (e.g. Sect. 1). We have used a recent compilation of Rakshit \& Stalin (2017), where they have matched the above parent sample of 11,101 NLSy1 galaxies with that of BLSy1 galaxies catalog both derived using SDSS DR-12. In their compilation, they found a sample of 5511 NLSy1 and BLSy1 galaxies, matching in the L-z plane (e.g. their figure 1 ). We noticed that out of our 223 NLSy1s, 149 (57 from $R O S A T$ and 92 from XMM-Newton) were indeed the member of this 5511 NLSy1s sample for which L-Z matched sample of 5511 BLSy1s exists.

However, due to limited X-ray observations of the aforementioned samples of NLSy1 and BLSy1 galaxies in ROSAT and XMM-Newton, we found it difficult to construct their exact L-Z matched sample for the X-ray analysis. Nonetheless, by restricting our search for X-ray observations of BLSy1s, only to the above sample of 5511 BLSy1s, we can expect to have a closely L-z match in the X-ray detected NLSy1s and BLSy1s samples. Therefore, we have cross-correlated these 5511 BLSy1s with that of the 2RXS catalog of ROSAT and also searched their any $X M M-N e w t o n$ telescope based observations using a similar procedure as we had adopted in the case of NLSy1 sample. The cross-correlation match in ROSAT resulted 

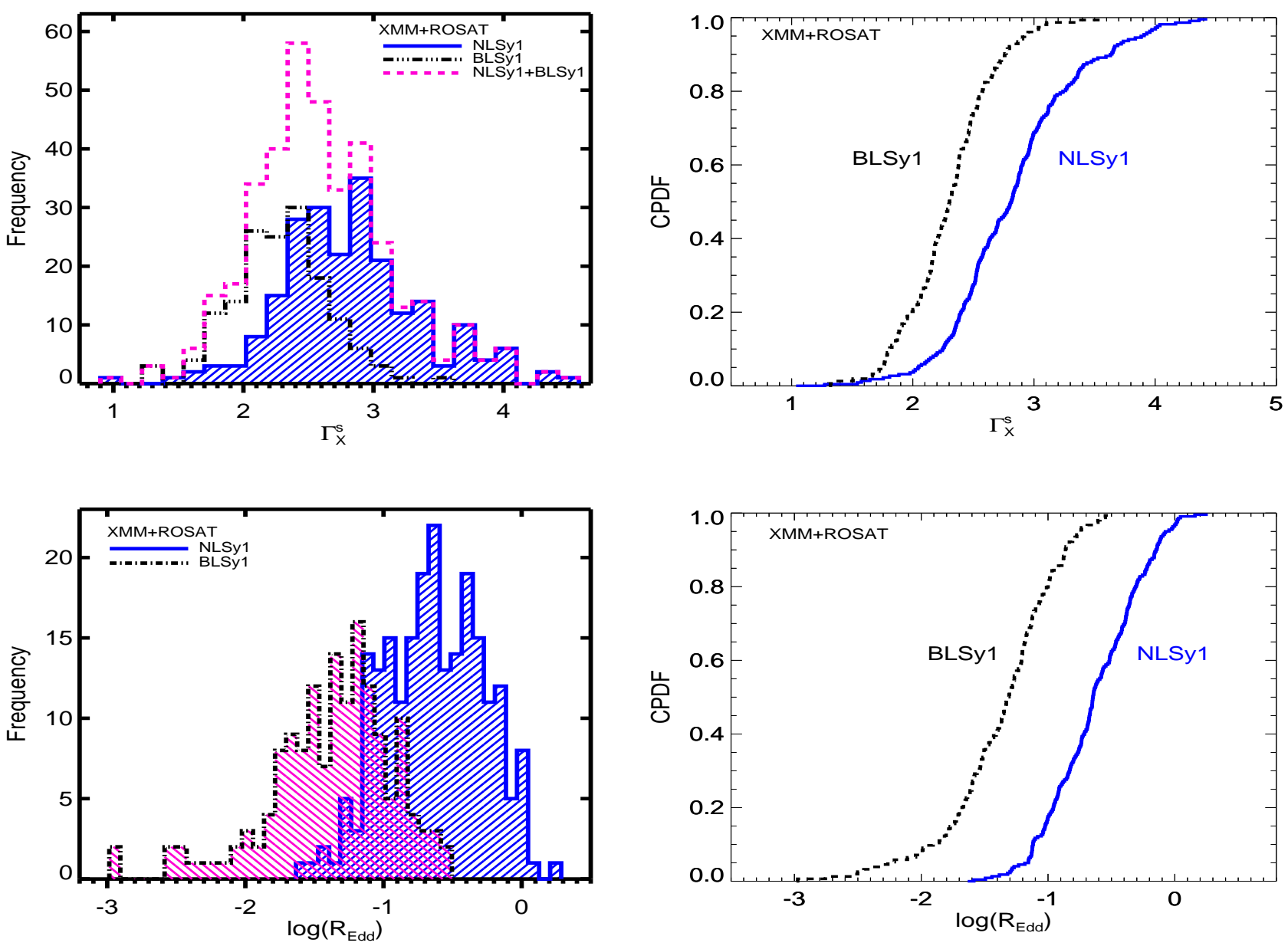

Fig. 3.- Top: Distribution of 0.1-2.0 keV soft X-ray photon indices (left) and its Cumulative probability distribution function (CPDF, right panel) for our combined ROSAT and XMM-Newton soft X-ray (0.1-2.0 keV) detected samples of 221 NLSy1s (blue, filled) and 154 BLSy1s (black, dashed with dotted line) along with joint [NLSy1+BLSy1] sample of 375 galaxies (pink, dashed). Bottom: The same as the top panels but for the Eddington ratios distribution (left, with blue filled for NLSy1s and pink filled for BLSy1s) and its CPDF (right), except for the joint [NLSy1+BLSy1] sample of 375 galaxies.

TABLE 1

Summary of the SAMPle SELECTION OF NLSy1 and BLSy1 GalaXies.

\begin{tabular}{|c|cc|cc|cc|}
\hline Telescope used & \multicolumn{5}{|c|}{ Selected (taken*) } \\
\hline & \multicolumn{2}{|c|}{ Soft energy sample } & Hard energy sample & Total $(0.3-10 \mathrm{keV})$ & energy sample \\
\hline & NLSy1 & BLSy1 & NLSy1 & BLSy1 & NLSy1 & BLSy1 \\
\hline ROSAT & $530(075)$ & $289(054)$ & -- & -- & -- & -- \\
\hline XMM-Newton & $697(146)$ & $332(100)$ & $148(53)$ & $103(46)$ & $148(139)$ & $103(97)$ \\
\hline BOTH $($ XMM+ROSAT) & $1227(221)$ & $621(154)$ & $148(53)$ & $103(46)$ & $148(139)$ & $103(97)$ \\
\hline
\end{tabular}

*After imposing a minimum $\mathrm{S} / \mathrm{N} \geq 10$ detection criterion (using 0.1-2.0 keV range in ROSAT and 0.3-10 keV in $X M M$ ) and counting the repeated sources only once (retaining only $X M M$ sources e.g. Sect. 2), along with the exclusion of those sources which could not be fitted with the adopted models (e.g. Sect. 4.2).

in 1156 BLSy1s, among them, 289 were covered by the PSPC instrument. Similarly, we found observations of 332 BLSy1s in XMM-Newton telescope. Further, we also applied the $\mathrm{S} / \mathrm{N} \geq 10$ detection criterion, as had also been used in the sample of NLSy1s (both for the ROSAT and $X M M$ subsamples). This resulted in a sample of 156 BLSy1s consisting of 54 sources from ROSAT and 103 from XMM-Newton. Further, we cross-correlated the 54 ROSAT BLSy1s with the 103 XMM BLSy1s, in order to check for any common sources among them, but none of the sources were found to be common.
Furthermore, it may be noted that we have used separately $X M M$-data for the X-ray analysis of total (0.3-10 $\mathrm{keV})$ and hard $(2-10 \mathrm{keV})$ energy bands as well. Out of 148 NLSy1s, the $\mathrm{S} / \mathrm{N} \geq 10$ criterion is fulfilled by $147 / 148$ in $0.3-10.0 \mathrm{keV}$ and $5 \overline{6} / 148$ in $2-10 \mathrm{keV}$. For BLSy1s, all qualify in total $0.3-10 \mathrm{keV}$ energy band, but only $51 / 103$ qualify this $\mathrm{S} / \mathrm{N} \geq 10$ criterion in hard energy band.

Further, reduction of the samples also occur due to non-convergence of the spectral fit (perhaps due to artifact in data, see Sect. 4.2), which in case of NLSy1s allow us to use $139 / 147$ in $0.3-10 \mathrm{keV}, 146 / 148$ in $0.1-2.0 \mathrm{keV}$, 

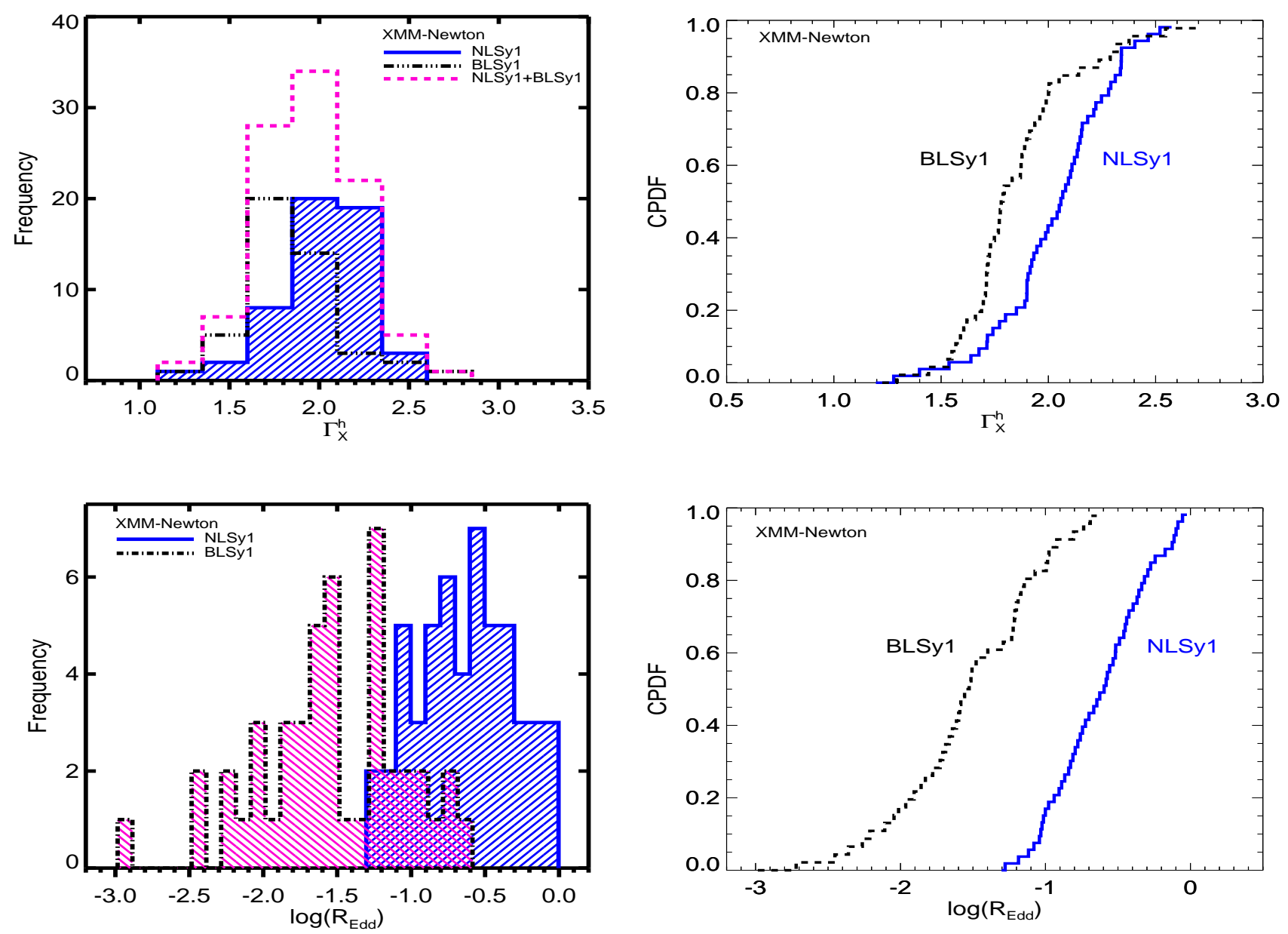

FIG. 4.- Same as Fig. 3, but using the XMM-Newton hard X-ray (2-10 keV) detected subsamples of 53 NLSy1 and 46 BLSy1 galaxies.

and $53 / 56$ in $2-10 \mathrm{keV}$. Similarly, for BLSy1s we could fit the $X M M$ sample of $97 / 103$ in $0.3-10 \mathrm{keV}, 100 / 103$ in $0.1-2.0 \mathrm{keV}$ and $46 / 51$ in $2-10 \mathrm{keV}$, as also summarized in Table 1. This led to our final samples of 221 NLSy1s (75 from the ROSAT and 146 from the XMM-Newton) and 154 BLSy1s (54 from the ROSAT and 100 from the $X M M-N e w t o n)$, for which we have shown the histograms of their redshift and luminosity in Fig. 1. As the figure shows, these two samples of NLSy1 and BLSy1 do moderately match in redshift, having the median redshifts of 0.21 and 0.26 , respectively. This gives the KolmogorovSmirnov test (K-S test) based probability of null hypothesis $\left(\mathrm{P}_{\text {null }}\right)$ of $\sim 3 \%$. However, the difference in luminosity is found to be much higher with median values of $\log \left(\lambda L_{\lambda}(5100 \AA)\right)[\mathrm{erg} / \mathrm{s} / \AA]$ of 43.67 and 44.08 , respectively, and $\mathrm{P}_{\text {null }}=2.43 \times 10^{-8}$.

\section{OBSERVATIONS AND DATA REDUCTION}

The X-ray data of our NLSy1 and BLSy1 galaxies were based on observations taken either with ROSAT/PSPC or with XMM-Newton/EPIC telescopes. The ROSAT 0.1-2.0 keV spectrum of each NLSy1, BLSy1 galaxy was extracted using the appropriate circular region around the source (e.g., see $3^{\text {rd }}$ para of Sect. 2). However, while extracting the corresponding background spectrum for a given source, we had ensured that its circular aperture is of the same size as taken for the source and is also in the vicinity of the source, free from any contamination from the other X-ray objects.

The standard XMM-Newton Science Analysis System (SAS) software package (version 16.1.0) was used in data reduction of $\mathrm{PN}$ detector of $X M M / E P I C$ with updated calibration files. EPCHAIN task was used on EPIC "Observation Data Files" for the preliminary processing. Calibrated and concatenated event lists were extracted using the EVSELECT task of SAS. We checked each source's data set for the high background proton flares by making its light curve in 10 to $12 \mathrm{keV}$ energy range which is used to make the good time interval (gti) file. Furthermore, pile up was also checked for each source's data set using the EPATPLOT task of SAS, with the appropriate circular region around the source, depending on the number of pixels containing the maximum flux of the source. If found, then that was removed by taking only the annulus region around the source for that data set. The SAS task ESPECGET was used to generate background and background corrected sources spectra. Further, it may be noted that we have used the $\chi^{2}$ minimization technique in our analysis for which essential criterion is that the data points included in this technique should be independent. So, while grouping our spectral data of XMM-Newton, we have taken care of this point and 

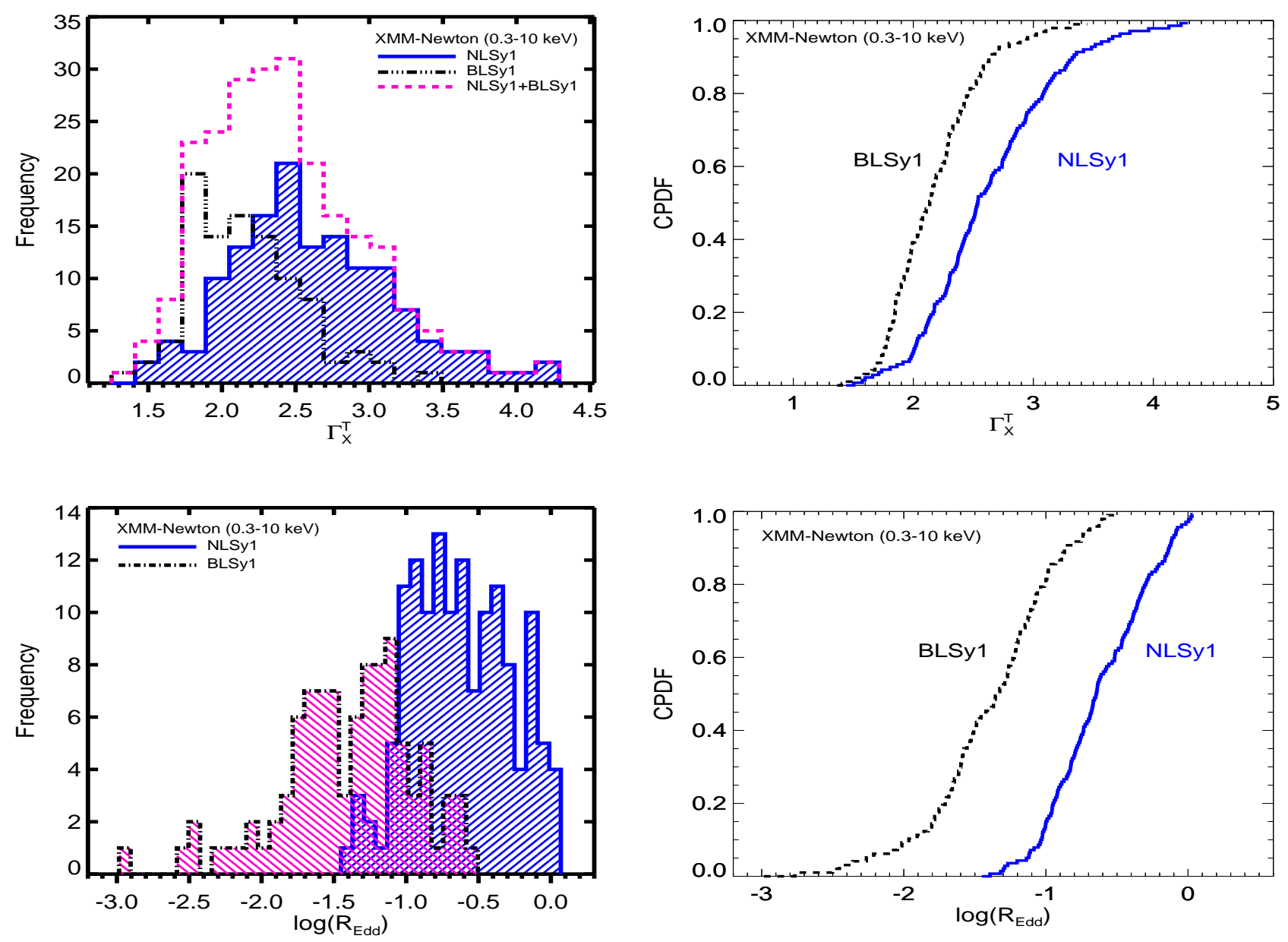

FIG. 5.- Same as Fig. 3, but using the XMM-Newton X-ray (0.3-10 keV) detected subsamples of 139 NLSy1 and 97 BLSy1 galaxies.

grouped each spectrum with a minimum of 20 counts subject to a condition that there should not be more than 4 bins per spectral resolution. This was done using the special task, SPECGROUP of SAS software.

\section{ANALYSIS}

\subsection{Black hole masses and Eddington ratios measurement}

To estimate the $M_{\mathrm{BH}}$ in a homogeneous way as pointed out in Sect. 1, we have opted to use the single epoch virial method, with improved virial empirical relation given by Vestergaard \& Peterson (2006) as;

$$
\begin{array}{r}
\log M_{\mathrm{B} H}=\log \left[\left(\frac{F W H M(H \beta)}{1000 k m s^{-1}}\right)^{2}\right]+(6.91 \pm 0.02) \\
+\log \left(\frac{\lambda L_{\lambda}(5100 \AA)}{10^{44} \text { erg s }^{-1}}\right)^{0.50 \pm 0.06}
\end{array}
$$

where $\mathrm{L}_{\lambda}(5100 \AA)$ is the monochromatic power-law continuum luminosity at $5100 \AA$ and $\operatorname{FWHM}(\mathrm{H} \beta)$ is the width of the broad component of $\mathrm{H} \beta$ line. We have taken both these parameters from the parent catalog of NLSy1s given by Rakshit et al. (2017). The procedure to obtain these parameters for BLSy1s was also similar to that used in NLSy1s, as outlined in Rakshit et al. (2017). In brief, in their method, they have first carried out a simultaneous fit of an AGN power-law continuum and host galaxy contribution, by masking the AGN emission lines. In the second step, a simultaneous fit on the host galaxy subtracted spectrum is carried out to optimize the best fit Gaussian profiles for the broad and narrow component of $\mathrm{H} \beta$ lines coming from the AGN broad and narrow line regions, respectively, along with the underneath local continuum and blends of Fe II emissions (e.g., see Rakshit et al. 2017).

Finally, for the estimations of the Eddington ratio, we have taken $\mathrm{L}_{\mathrm{bol}}=9.8 \times \lambda L_{\lambda}(5100 \AA)$ (McLure \& Dunlop $2004)$ and $\mathrm{L}_{\mathrm{E} d d}=1.45 \times 10^{38}\left(M_{\mathrm{B} H} / M_{\odot}\right) \mathrm{erg} \mathrm{s}^{-1}$, assuming a mixture of hydrogen and helium so that the mean molecular weight is $\mu=1.15$. The values of $\log \left(\mathrm{M}_{\mathrm{B} H}\right)$ and $\log \left(\mathrm{R}_{\mathrm{E} d d}\right)$ along with $\Gamma_{X}^{h}, \Gamma_{X}^{s}$, and $\Gamma_{X}^{T}$ for each NLSy1 and BLSy1 galaxy are given in columns $6,7,8$, 11, and 14 of Table 3 and Table 4, respectively.

\subsection{X-ray spectral analysis}

For the spectral analysis of ROSAT detected 75 NLSy1 and 54 BLSy1 galaxies, we have used XSPEC version 12.10.1 (Arnaud 1996; Dorman \& Arnaud 2001) tasks of HEASOFT. The response matrices files (RMFs) required 

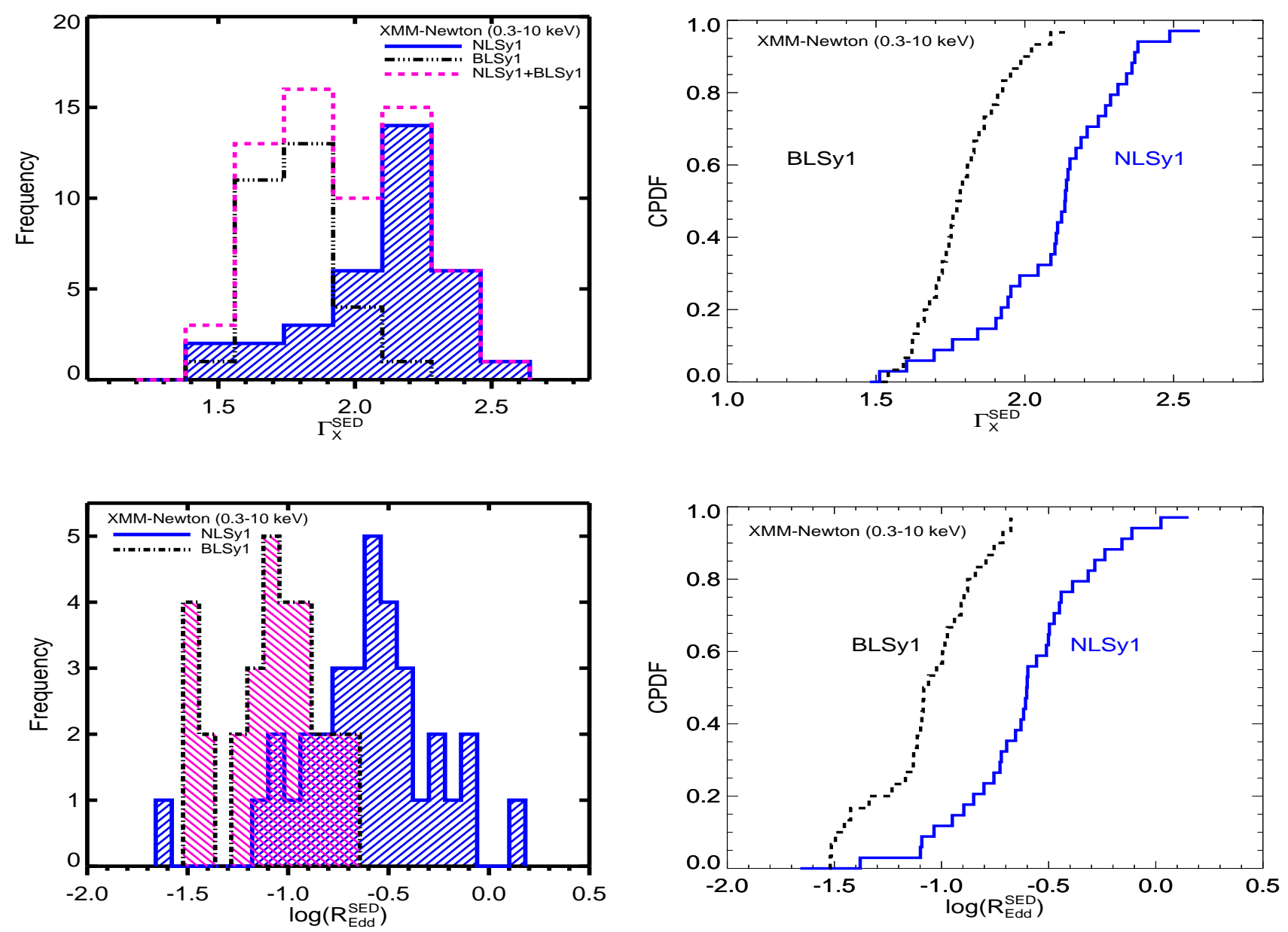

FIG. 6.- Same as Fig. 3, but using the X-ray hot photon indices $\left(\Gamma_{X}^{\text {hot }}\right)$ and Eddington ratios $\left(\mathrm{R}_{\mathrm{E} d d}^{\mathrm{SED}}\right)$ of 34 NLSy1 and 30 BLSy1 galaxies based on the AGNSED model (Kubota \& Done 2018) in the 0.3-10 keV energy band of the XMM-Newton data.

TABLE 2

Summary of the BeSt fit MOdel USED for the SPECTRAl Fitting of NLSy1 and BLSy1 Galaxies.

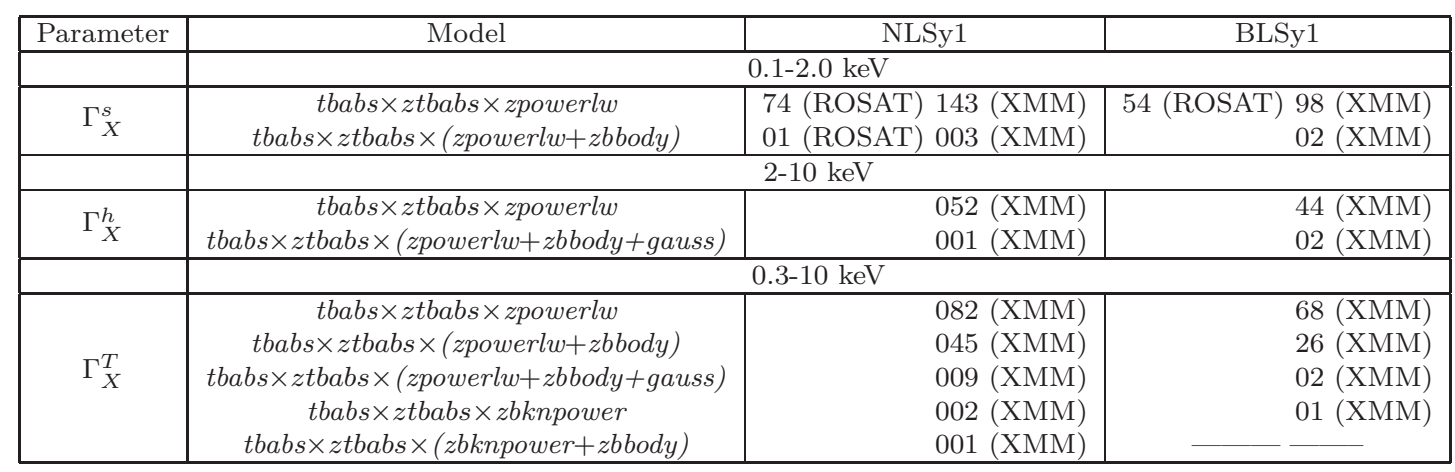

for the spectral fitting were obtained from the latest calibration database available publicly on the HEASARC calibration database ${ }^{2}$ and the ancillary response files (ARFs) were generated with the PCARF task of HEASOFT. The extracted spectrum (e.g. Sect. 3) of each NLSy1, BLSy1 was grouped with a minimum of 20 counts per bin using the GRPPHA routine of the XSELECT task,

\footnotetext{
2 https://heasarc.gsfc.nasa.gov/docs/heasarc/caldb/
}

which permitted us to use $\chi^{2}$ minimization for spectral fitting. To obtain the soft X-ray $(0.1-2.0 \mathrm{keV})$ photon indices (hereafter $\Gamma_{X}^{s}$ ), we have used the physically motivated model consisting of basic power law and the double neutral absorption (i.e., tbabs $\times$ ztbabs $\times z$ powerlw) in XSPEC software to the spectral data in the observed frame energy range of 0.1-2.0 keV. Special cares were taken to properly fit the absorption of soft X-rays during the fitting of NLSy1 and BLSy1 galaxies. During 
TABLE 3

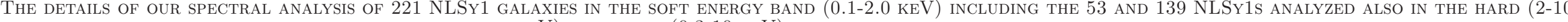
KEV) AND TOTAL (0.3-10 KEV) ENERGY BANDS, RESPECTIVELY.

\begin{tabular}{|c|c|c|c|c|c|c|c|c|c|c|c|c|c|c|c|c|c|c|c|c|}
\hline Source Name & $\begin{array}{l}{ }^{\mathrm{RA}} \\
(\mathrm{deg})\end{array}$ & $\begin{array}{l}\mathrm{DEC} \\
\text { (deg) }\end{array}$ & ${ }_{\text {(redshift) }}^{z_{e m}}$ & $\begin{array}{c}\mathrm{S} / \mathrm{N} \\
(0.3-10 \mathrm{keV})\end{array}$ & $\log \mathrm{M}_{\mathrm{B} H}$ & $\log \mathrm{R}_{\mathrm{E} d d}$ & $\Gamma_{x}^{h}$ & $\begin{array}{c}\delta \Gamma_{x}^{h-} \\
(2-10 \mathrm{keV})\end{array}$ & $\delta \Gamma_{x}^{h+}$ & $\Gamma_{X}^{s}$ & $\begin{array}{c}\delta \Gamma_{X}^{s-} \\
(0.1-2.0 \mathrm{keV})\end{array}$ & $\delta \Gamma_{X}^{s+}$ & $\Gamma_{x}^{T}$ & $\begin{array}{c}\delta \Gamma_{x}^{T-} \\
(0.3-10 \mathrm{keV})\end{array}$ & $\delta \Gamma_{X}^{T+}$ & $\begin{array}{c}\log \left(\lambda L_{\lambda}\right) \\
(5100 \AA) \\
\operatorname{erg~s}^{-1}\end{array}$ & $\begin{array}{c}\mathrm{FWHM}(\mathrm{H} \beta) \\
\mathrm{km} \mathrm{s}^{-1}\end{array}$ & $\begin{array}{c}\delta(F W H M(H \beta)) \\
\mathrm{km} \mathrm{s}^{-1}\end{array}$ & $\begin{array}{c}\text { Aperture } \\
\text { used } \\
\text { (arsec) }\end{array}$ & $\begin{array}{l}\text { Telescope } \\
\text { used }\end{array}$ \\
\hline (1) & (2) & (3) & (4) & (5) & (6) & (7) & (8) & (9) & (10) & (11) & (12) & (13) & (14) & (15) & (16) & (17) & (18) & (19) & (20) & (21) \\
\hline J010712.00+140845.6 & 016.800 & 14.146 & 0.0767 & 054.0 & 6.0221 & -0.6422 & 2.0618 & -0.2783 & 0.5270 & 2.5856 & -0.1670 & 0.2440 & 2.4017 & -0.0501 & 0.0797 & 42.55 & 829 & 29 & 12.59 & $X M M$ \\
\hline J014644.88-004044.4 & 026.687 & -00.679 & 0.0824 & 030.2 & 6.7026 & -0.6527 & -- & -- & - & 2.9201 & -0.2971 & 0.3178 & - & -- & -- & 43.22 & 1234 & 20 & 57.25 & ROSAT \\
\hline$J 081442.00+212916.8$ & 123.675 & 21.488 & 0.1626 & 175.6 & 7.3140 & -0.4641 & 2.0722 & -0.0711 & 0.1415 & 2.7822 & -0.0454 & 0.0715 & 2.8400 & -0.0875 & 0.1012 & 44.02 & 1574 & 22 & 35.87 & $X M M$ \\
\hline & & & $\ldots .$. & & & $\ldots$ & & & $\ldots .$. & & & & & 2 & & $\ldots$ & ‥ & & & \\
\hline
\end{tabular}

Note: - The entire table is available in the online version. Only a portion of this table is shown here, to display its form and contents.

TABLE 4

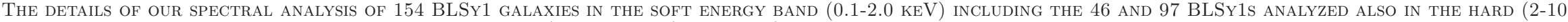
KEV) AND TOTAL (0.3-10 KEV) ENERGY BANDS, RESPECTIVELY.

\begin{tabular}{|c|c|c|c|c|c|c|c|c|c|c|c|c|c|c|c|c|c|c|c|c|}
\hline Source Name & $\begin{array}{l}\mathrm{RA} \\
(\mathrm{deg})\end{array}$ & $\begin{array}{l}\mathrm{DEC} \\
(\mathrm{deg})\end{array}$ & $\begin{array}{c}z_{e m} \\
\text { (redshift) }\end{array}$ & $\mathrm{S} / \mathrm{N}$ & $\log \mathrm{M}_{\mathrm{B} H}$ & $\log \mathrm{R}_{\mathrm{E} d d}$ & $\Gamma_{X}^{h}$ & $\begin{array}{c}\delta \Gamma_{X}^{h-} \\
(2-10 \mathrm{keV})\end{array}$ & $\delta \Gamma_{x}^{h+}$ & $\Gamma_{X}^{s}$ & $\begin{array}{c}\delta \Gamma_{X}^{s-} \\
(0.1-2.0 \mathrm{keV})\end{array}$ & $\delta \Gamma_{X}^{s+}$ & $\Gamma_{x}^{T}$ & $\begin{array}{c}\delta \Gamma_{X}^{T-} \\
(0.3-10 \mathrm{keV})\end{array}$ & $\delta \Gamma_{x}^{T+}$ & $\begin{array}{c}\log \left(\lambda L_{\lambda}\right) \\
(5100 \AA) \\
\operatorname{erg~s}^{-1}\end{array}$ & $\begin{array}{c}\text { FWHM(H } \beta) \\
\mathrm{km} \mathrm{s}^{-1}\end{array}$ & $\begin{array}{c}\delta(F W H M(H \beta)) \\
\mathrm{km} \mathrm{s}^{-1}\end{array}$ & $\begin{array}{c}\text { Aperture } \\
\text { used } \\
(\operatorname{arcsec})\end{array}$ & $\begin{array}{l}\text { Telescope } \\
\text { used }\end{array}$ \\
\hline (1) & (2) & (3) & (4) & (5) & (6) & (7) & (8) & (9) & (10) & (11) & (12) & (13) & (14) & (15) & (16) & (17) & (18) & (19) & (20) & (21) \\
\hline J002113.20-020115.6 & 005.305 & -02.021 & 0.7621 & 024.6 & 8.8620 & -1.5821 & -- & -- & -- & 1.8349 & -0.2342 & 0.4626 & 1.9144 & -0.1726 & 0.2121 & 44.45 & 7303 & 733 & 16.45 & $X M M$ \\
\hline J044759.52-043231.2 & 071.998 & -04.542 & 0.2569 & 016.2 & 8.5913 & -1.6014 & -- & -- & -- & 2.7118 & -0.1975 & 0.3120 & -- & -- & -- & 44.16 & 6318 & 131 & 93.79 & ROSAT \\
\hline J075112.24+174351.6 & 117.801 & 17.731 & 0.1861 & 169.6 & 7.9220 & -1.1421 & 1.7954 & -0.0854 & 0.0580 & 2.2096 & -0.0355 & 0.0711 & 2.1931 & -0.0185 & 0.0595 & 43.95 & 3299 & 54 & 22.77 & $X M M$ \\
\hline
\end{tabular}

Note: - The entire table is available in the online version. Only a portion of this table is shown here, to display its form and contents. 
the fitting, the redshift of the source was kept fixed to its precise value known from its optical spectrum and also Galactic hydrogen column density in the direction of the source was kept fixed based on the value given by Dickey \& Lockman (1990). However, the normalization, host galaxy absorption, intrinsic absorption, and $\Gamma_{X}^{s}$ of the source were the free parameters of the fit. In one of our NLSy1 galaxy, viz., J162901.20+400758.8, we noted soft X-ray excess below $0.5 \mathrm{keV}$. For fitting this source, we added a blackbody component to our basic model (i.e., tbabs $\times z t b a b s \times(z p o w e r l w+z b b o d y))$. In summary, we could estimate using ROSAT data in 0.1-2.0 $\mathrm{keV}$ range, the $\Gamma_{X}^{s}$ of 75 NLSy1 and 54 BLSy1 galaxies, as listed in Table 3 and Table 4 (see columns 11-13), respectively.

For spectral analysis of the $X M M / E P I C-P N$ sample, we have used physically motivated models as had been employed above for $R O S A T / P S P C$ sample. Our model fittings converged well to estimate soft X-ray (0.3$2.0 \mathrm{keV}$ ) photon indices for the 146/148 NLSy1 and 100/103 BLSy1 galaxies, and for the hard X-ray (2-10 $\mathrm{keV}$ ) photon indices (hereafter $\Gamma_{X}^{h}$ ) in 53/56 NLSy1 and 46/51 BLSy1 galaxies. Among them, 51 NLSy1 and 44 BLSy1 galaxies were fitted in both hard and soft energy bands of XMM-Newton, while 2 NLSy1 and 2 BLSy1 galaxies were only fitted in hard X-ray energy band. We also noticed the soft X-ray excesses in 3 NLSy1 and 2 BLSy1 galaxies and fitted them by adding black body component to our basic model. However, one NLSy1 galaxy (viz., J105128.32+335851.6) and 2 BLSy1 (viz., J125553.04+272403.6 and J161745.6+060350.4) galaxies also showed emission line feature. Therefore, to fit these sources, we had added the black body and Gaussian components to our basic model, viz., tbabs $\times z t b a b s \times(z p o w e r l w+z b b o d y+$ gauss $)$. A summary of these best fit models is given in Table 2 .

Representative data and best fit folded models for one member of NLSy1 and BLSy1 galaxies samples, each in ROSAT and XMM-Newton are shown in the top and bottom panels of Fig. 2, respectively. The typical range of the X-ray photon index varies for our samples of NLSy1 and BLSy1 galaxies in the soft energy band from 1.1-4.4 and 1.3-3.6, respectively, while in the hard energy band varies from 1.2-2.6 and 1.2-2.7, respectively.

In summary, based on our combined sample of 223 NLSy1s (75 from the ROSAT and 148 from the XMM), we got $\Gamma_{X}^{s}$ measurements for the 221 NLSy1s (75 from the ROSAT and 146 from the $X M M$ ) as listed in Table 3. Similarly, out of the sample of 156 BLSy1s (54 from the ROSAT and 103 from the $X M M)$, we got $\Gamma_{X}^{s}$ for 154 BLSyls (54 from the ROSAT and 100 from the $X M M)$ as listed in Table 4 . The histograms and cumulative probability distribution functions (CPDFs) of $\Gamma_{X}^{s}$ for the samples of 221 NLSy1 and 154 BLSy1 galaxies along with their combined sample (hereafter [NLSy1+BLSy1]) are shown in the top panels of Fig. 3. However, for the $\Gamma_{X}^{h}$ measurements, we could use only 53 NLSy1 and 46 BLSy1 galaxies based on their XMM subsamples of 56 NLSy1 and 51 BLSy1 galaxies, as listed in Table 3 and Table 4, respectively. The histograms and CPDFs of $\Gamma_{X}^{h}$ are shown in the top panels of Fig. 4.

\subsection{X-ray spectral analysis in 0.3-10 keV energy range}

The spectral coverage of the $X M M$ data also allows us to estimate the $0.3-10 \mathrm{keV}$ photon indices (hereafter $\Gamma_{X}^{T}$ ). This is also useful to compare $\Gamma_{X}^{T}$ distribution with the $\Gamma_{X}^{s}$ and $\Gamma_{X}^{h}$ distributions, both for NLSy1 and BLSy1 galaxies. Our fittings in the $0.3-10 \mathrm{keV}$ could converge for 82/146 NLSy1s and 68/100 BLSy1s with our basic model, i.e., tbabs $\times z$ tbabs $\times(z p o w e r l w)$. However, in case of 45 NLSy1 and 26 BLSy1 galaxies, we had to add black body component to our basic model, i.e., tbabs $\times z t b a b s \times(z p o w e r l w+z b b o d y)$. There were requirement of an additional Gaussian emission component for the fittings of 8 NLSy1 and 2 BLSy1 galaxies., i.e., tbabs $\times z$ tbabs $\times(z$ powerlw+zbbody+gauss $)$. For 2 NLSy 1 s (viz., J150506.48+032631.2, J151312.48+001937.2) and 1 BLSy1 (viz., J135435.76+180516.8), it was found that they can not be fitted with single powerlaw, therefore, they were accounted by broken power law model i.e., tbabs $\times z$ tbabs $\times(z b k n p o w e r)$. For one NLSy1, viz., J12410.56+331702.4, similar feature with additional soft X-ray excess was accommodated by the inclusion of black-body emission, e.g., as tbabs $\times z t b a b s \times(z b k n p o w e r+z b b o d y)$. The above combination of models, as also summarized in Table 2, have allowed us to estimate $\Gamma_{X}^{\mathrm{T}}$ of $139 / 148$ NLSy1 and $97 / 100$ BLSy1 galaxies, whose distributions are shown in Fig. 5. From this distributions, it can be seen that the typical range of $\Gamma_{X}^{\mathrm{T}}$ varies for our subsamples of NLSy1 and BLSy1 galaxies in the total energy band from 1.4-4.3 and 1.4-3.4, respectively which is consistent with the range $\Gamma_{X}^{\mathrm{s}}$ and $\Gamma_{X}^{\mathrm{h}}$ (e.g see Sect. 4.2 above).

\subsection{X-ray spectral analysis in 0.3-10 keV energy range using AGNSED model}

Another physically motivated model consisting of the spectral energy distribution of the AGN (hereafter, $A G N S E D$, or 'agnsed', Kubota \& Done 2018) and the Galactic absorption (i.e., tbabs $\times$ agnsed), can also be employed using XSPEC on the $X M M$ spectra in $0.3-10 \mathrm{keV}$ energy range. Here, we have limited ourself only to those 53 NLSy1s and 46 BLSy1s XMM sources, for which their hard energy data has also enabled us (due to sufficient $\mathrm{S} / \mathrm{N}$ ) to estimate their $\Gamma_{X}^{h}$ as well (e.g. Sect. 4.2). Special cares were also taken to properly fit the absorption of soft X-rays, by fixing the Galactic hydrogen column density to its value given by Dickey \& Lockman (1990). During the fitting black hole mass, redshift, and comoving (proper) distance of the source were kept fixed to their precise value known from the optical spectra. We also kept fix, the black hole spin, inclination angle $\mathrm{i}$ (for the warm comptonising component and the outer disc), electron temperature (for the hot comptonization component), reprocessing, and normalization parameters to $0.5,30^{\circ}, 100 \mathrm{keV}, 0$, and 1 , respectively. However, the other parameters of this model, viz., Eddington ratio $\left(\mathrm{R}_{\mathrm{E} d d}^{\mathrm{S} E D}=\dot{m}\right)$, electron temperature for the warm comptonization component ( $\left.\mathrm{kT}_{e}^{\text {warm }}\right)$, hot photon index $\left(\Gamma_{X}^{\text {hot }}\right)$, warm photon index $\left(\Gamma_{X}^{\text {warm }}\right)$, outer radius of the hot comptonization component, and outer radius of the warm comptonization component were kept free.

In 3 NLSy1 and 2 BLSy1 galaxies, we noted soft Xray feature or warm absorption below $2 \mathrm{keV}$. To carry out the fitting of these 5 sources, we considered a warm absorber model, namely zxipcf in addition to the above 
model (i.e., tbabs $\times z x i p c f \times a g n s e d)$. The above combination of models allowed us to estimate $\Gamma_{X}^{\text {hot }}$ and $\mathrm{R}_{\mathrm{E} d d}^{\mathrm{SED}}$ of 34 NLSy1 and 30 BLSy1 galaxies, whose distributions are shown in Fig. 6. However, the fit of the remaining 19 (out of a total of 53) NLSy1 and 16 BLSy1 (out of a total of 46) galaxies did not converge with either of the above models due to either their bad data coverage up to $10 \mathrm{keV}$ or non-convergence of their fit parameters.

\section{RESULTS}

\subsection{Comparison of $\Gamma_{X}$ and $R_{\mathrm{E} d d}$ among the samples of BLSy1 and NLSy1 galaxies}

We used a sample of 221 NLSy1s to compare its physical parameters with 154 BLSy1s (e.g., see Table 1, Sect. 2) moderately matching in the redshift plane (e.g., see Fig. 1). The histograms of our homogeneous analysis (e.g. Sect. 4.2) of 0.1-2.0 keV photon indices for both the samples are shown in the top left panel of Fig. 3. As can be seen from these histogram plots that there is a clear difference in the photon indices distributions with the median values of 2.81 and 2.30 for the samples of NLSy1 and BLSy1 galaxies, respectively, with former being systematically steeper. This is also evident from the CPDF plots of $\Gamma_{X}^{s}$ as shown in the top right panel of Fig. 3. To quantify this difference statistically, we have carried out the Kolmogorov-Smirnov test (K-S test) giving the probability of the null hypothesis (i.e., two distribution are similar) as $\mathrm{P}_{\text {null }}=4.02 \times 10^{-19}$, suggesting a clear significant difference. Similarly, we have also plotted distributions of $\mathrm{R}_{\mathrm{E} d d}$ for both NLSy1 and BLSy1 galaxies in the bottom panels of Fig. 3. As can be seen from its histograms (bottom left panel) and CPDFs (bottom right panel) that $\mathrm{R}_{\mathrm{E} d d}$ of the sample of 221 NLSy1s is systematically higher as compare to the sample of 154 BLSy1s, with the median values of 0.23 and 0.05 , respectively, resulting in K-S test based $P_{\text {null }}$ of $2.66 \times 10^{-35}$.

Furthermore, we also did the above comparison in the hard energy band (2-10 keV) using the $\Gamma_{X}^{h}$ estimated for the subsamples of 53 NLSy1 and 46 BLSy1 galaxies with their distributions as shown in the top left panel of Fig. 4. The $\Gamma_{X}^{h}$ for the subsamples of NLSy1 and BLSy1 galaxies having the median values of 2.06 and 1.78 , respectively, and the K-S test based $P_{\text {null }}$ of $5.13 \times 10^{-5}$, suggesting a smaller difference in their photon indices in comparison to the difference found in the soft energy band.

Additionally, we also carried out a similar comparison in the total energy band $(0.3-10 \mathrm{keV})$ using the $\Gamma_{X}^{T}$ of 139 NLSy1 and 97 BLSy1 galaxies with their distributions as shown in the top left panel of Fig. 5. The median values of $\Gamma_{X}^{T}$ for the subsamples of NLSy1 and BLSy1 galaxies are found to be 2.53 and 2.13 , respectively, resulting in K-S test based $P_{\text {null }}$ of $4.50 \times 10^{-9}$. This still suggests a significant difference in their photon indices though it is smaller than the difference found in the soft energy band.

Furthermore, quantification of any such physical differences among NLSy1 and BLSy1 galaxies can also be obtained based on the correlations of spectral indices (in the soft hard and total $0.3-10 \mathrm{keV}$ X-ray energy bands) with the other parameters of nuclear activities of AGNs such as $\mathrm{R}_{\mathrm{E} d d}$, $\mathrm{L}_{\mathrm{bol}}, \mathrm{M}_{\mathrm{B} H}$, and $\mathrm{FWHM}(\mathrm{H} \beta)$, as we discuss in next subsection.

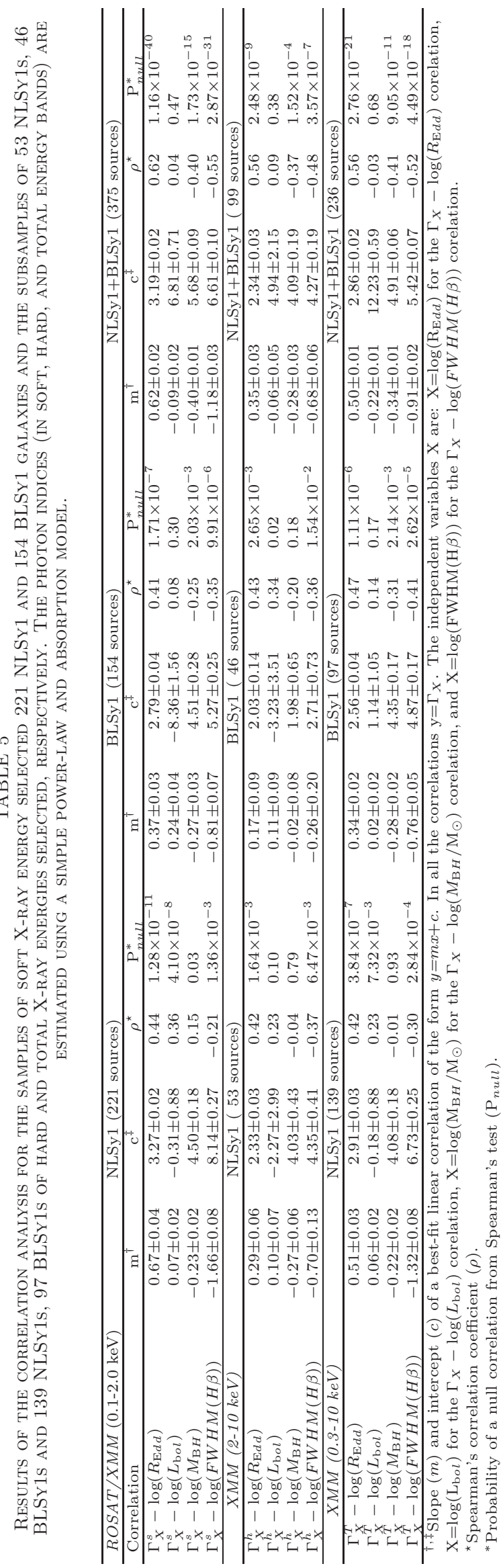


TABLE 6

Results of the Correlation analysis in $0.3-10 \mathrm{kEV}$ For the SAmples of 34 NLSy1 AND 30 BLSy1 Galaxies using the AGNSED MODEL.

\begin{tabular}{|c|c|c|c|c|c|c|c|c|}
\hline$X M M(0.3-10.0 \mathrm{keV})$ & \multicolumn{3}{|c|}{ NLSy1 (34 sources) } & \multicolumn{5}{|c|}{ BLSy1 (30 sources) } \\
\hline Correlation $^{\dagger}$ & $\mathrm{m}$ & $\mathrm{c}$ & $\rho$ & $\overline{\mathrm{P}_{\text {null }}}$ & $\mathrm{m}$ & $\mathrm{c}$ & $\rho$ & $\mathrm{P}_{\text {null }}$ \\
\hline$\Gamma_{X}^{\text {hot }}-\log \left(R_{\mathrm{E} d d}^{\mathrm{SED}}\right)$ & $0.44 \pm 0.09$ & $2.42 \pm 0.04$ & 0.62 & $9.71 \times 10^{-5}$ & $0.40 \pm 0.11$ & $2.22 \pm 0.11$ & 0.54 & $1.87 \times 10^{-3}$ \\
\hline$\Gamma_{X}^{\text {hot }}-\log \left(L_{\mathrm{bol}}\right)$ & $0.13 \pm 0.04$ & $-3.74 \pm 1.83$ & 0.42 & $1.42 \times 10^{-2}$ & $0.10 \pm 0.04$ & $-2.92 \pm 1.77$ & 0.53 & $2.40 \times 10^{-3}$ \\
\hline$\Gamma_{X}^{\text {hot }}-\log \left(M_{\mathrm{B} H}\right)$ & $-0.07 \pm 0.03$ & $2.69 \pm 0.24$ & 0.16 & $3.73 \times 10^{-1}$ & $0.04 \pm 0.03$ & $-2.08 \pm 0.28$ & 0.01 & $9.47 \times 10^{-1}$ \\
\hline$\Gamma_{X}^{\text {hot }}-\log (F W H M(H \beta))$ & $-0.48 \pm 0.10$ & $3.65 \pm 0.30$ & -0.18 & $3.03 \times 10^{-1}$ & $-0.24 \pm 0.08$ & $2.67 \pm 0.32$ & -0.22 & $2.49 \times 10^{-1}$ \\
\hline $\log \left(R_{\mathrm{E} d d}^{\mathrm{SED}}\right)-\log \left(R_{\mathrm{E} d d}\right)$ & $0.30 \pm 0.10$ & $-0.42 \pm 0.02$ & 0.47 & $2.44 \times 10^{-3}$ & $0.14 \pm 0.12$ & $-0.82 \pm 0.20$ & 0.51 & $7.28 \times 10^{-4}$ \\
\hline
\end{tabular}

${ }^{\dagger}$ The correlation parameters are the same as Table 5 , but in $y=m x+c$ fit, for $\mathrm{y}=\Gamma_{X}^{\text {hot }}$ in all the correlations and $\mathrm{X}=\log \left(R_{\mathrm{E} d d}^{\mathrm{SE} D}\right)$ in the $\Gamma_{X}^{\text {hot }}-\log \left(R_{\mathrm{E} d d}^{\mathrm{SED}}\right)$ correlation. In the last row $\mathrm{y}$ and $\mathrm{x}$ are $\log \left(R_{\mathrm{E} d d}^{\mathrm{S} E D}\right)$ and $\log \left(R_{\mathrm{E} d d}\right)$, respectively.

\subsection{Comparison of $\Gamma_{X}$ correlations with $A G N$ parameters among the samples of NLSY1 and BLSy1 galaxies}

Results based on our correlations analysis for the samples of 221 NLSy1s, 154 BLSy1s and their combined sample (i.e., 375 [NLSy1+BLSy1]) are shown in Fig. 7. This figure shows the plots of $\mathrm{R}_{\mathrm{E} d d}, \mathrm{~L}_{\mathrm{bol}}, \mathrm{M}_{\mathrm{BH}}$ and $\operatorname{FWHM}(\mathrm{H} \beta)$ versus $\Gamma_{X}^{s}$ for NLSy1, BLSy1 and [NLSy1+BLSy1] galaxies in the left, middle and right panels, respectively. The statistical quantifications of correlations of these parameters with $\Gamma_{X}^{s}$ are summarised in Table 5. As can be seen from the top panels of Fig. 7 that the positive correlations between $\Gamma_{X}^{s}$ and $\log \left(\mathrm{R}_{\mathrm{E} d d}\right)$ are quite apparent for the samples of NLSy1 (top left panel), BLSy1 (top middle panel), and [NLSy1+BLSy1] (top right panel) galaxies, respectively. We have also quantified these correlations with the fitting function of the form $y=m x+c$ by standard $\chi^{2}$ - minimization method which yields the relations, for the sample of NLSy1s as

$$
\Gamma_{X}^{s}=(0.67 \pm 0.04) \log \left(R_{\mathrm{E} d d}\right)+(3.27 \pm 0.02)
$$

, for the sample of BLSy1s as

$$
\Gamma_{X}^{s}=(0.37 \pm 0.03) \log \left(R_{\mathrm{E} d d}\right)+(2.79 \pm 0.04)
$$

and for the joint sample of NLSy1 and BLSy1 galaxies as

$$
\Gamma_{X}^{s}=(0.62 \pm 0.02) \log \left(R_{\mathrm{E} d d}\right)+(3.19 \pm 0.02)
$$

as shown by the solid red line in the plots of $\Gamma_{X}^{s}-$ $\log \left(R_{\mathrm{E} d d}\right)$ in the top panels of Fig. 7. This very good correlation is also supported based on their Spearman's rank correlation coefficient $(\rho)$ of $0.44,0.41$ and 0.62 with the probability of null correlation $\left(\mathrm{P}_{\text {null }}\right)$ of $1.28 \times 10^{-11}$, $1.71 \times 10^{-7}$, and $1.16 \times 10^{-40}$ for the samples of NLSy1, BLSy1 and [NLSy1+BLSy1] galaxies. We note here that the correlation coefficients found for the samples of NLSy1 and BLSy1 galaxies are almost similar, however, the difference is significant in the slopes of their $\Gamma_{X}^{s}-\log \left(R_{\mathrm{E} d d}\right)$ linear fit, with $m=0.67 \pm 0.04$ and $0.37 \pm 0.03$, respectively. Our above correlations between $\Gamma_{X}^{s}$ and $\log \left(\mathrm{R}_{\mathrm{E} d d}\right)$ give hint that $\Gamma_{X}^{s} \propto L_{\mathrm{bol}} / L_{\mathrm{E} d d}$ which implies that $\Gamma_{X}^{s} \propto L_{\mathrm{bol}}$ and $\Gamma_{X}^{s} \propto L_{\mathrm{E} d d}^{-1}$. As we know that $\mathrm{L}_{\mathrm{E} d d} \propto M_{\mathrm{B} H}$ and $\mathrm{M}_{\mathrm{B} H} \propto(F W H M(H \beta))^{2}$, there- fore, a very good $\Gamma_{X}^{s}-\log \left(R_{\mathrm{E} d d}\right)$ correlation can be due to intrinsic $\Gamma_{X}^{s}-\log \left(L_{\mathrm{bol}}\right)$ and $\Gamma_{X}^{s}-\log \left(M_{\mathrm{B} H}^{-1}\right)$ correlations or due to both these correlations. We tested these possibilities in the samples of NLSy1, BLSy1 and [NLSy1+BLSy1] galaxies. We find a significant $\Gamma_{X}^{s}-\log \left(L_{\mathrm{bol}}\right)$ correlation for the sample of NLSy1s with $\rho=0.36, \mathrm{P}_{\text {null }}=4.10 \times 10^{-8}$. The corresponding correlations are found to be nonsignificant for the samples of BLSy1 (with $\rho=0.08 \& \mathrm{P}_{\text {null }}=0.30$ ) and $\left[\right.$ NLSy1+BLSy1] galaxies (with $\rho=0.04 \& \mathrm{P}_{\text {null }}=0.47$ ). However, for the $\Gamma_{X}^{s}-\log \left(M_{\mathrm{B} H}\right)$, we found a good anticorrelation in the joint sample of NLSy1 and BLSy1 galaxies (with $\rho=-0.40 \& \mathrm{P}_{\text {null }}=1.73 \times 10^{-15}$ ), though it was found to be nonsignificant when the samples of NLSy1 and BLSy1 galaxies considered separately.

On the other hand, the significant $\Gamma_{X}^{s}-$ $\log (F W H M(H \beta))$ anticorrelations are found for the samples of BLSy1 (with $\rho=-0.35 \&$ $\left.\mathrm{P}_{\text {null }}=9.91 \times 10^{-6}\right)$ and $[\mathrm{NLSy} 1+\mathrm{BLSy} 1]$ galaxies (with $\rho=-0.55 \& \mathrm{P}_{\text {null }}=2.87 \times 10^{-31}$ ). However, this correlation is found to be nominal for the sample of NLSy1s with $\rho=-0.21$ and $\mathrm{P}_{\text {null }}=1.36 \times 10^{-3}$. Summary of all the above correlations along with $\rho$ and $\mathrm{P}_{\text {null }}$ values separately for the NLSy1s, BLSy1s, and their combined samples is given in Table 5 .

It may be noted here that the effect of soft X-ray excess, cold absorbers, warm absorbers, and other low energy spectral complexities are generally prominent below $2 \mathrm{keV}$ (Brandt et al. 1997) in the NLSy1 and BLSy1 galaxies. So, to confirm the aforementioned correlations, found for the samples of NLSy1 and BLSy1 galaxies between $\Gamma_{X}^{s}$ and $\log \left(\mathrm{R}_{\mathrm{E} d d}\right)$, we analyzed $\Gamma_{X}^{h}-\log \left(R_{\mathrm{E} d d}\right)$ correlation in hard energy $(2-10 \mathrm{keV})$ band which is thought to be probably less affected by soft X-ray excess. For this, we analyzed 53 NLSy1 and 46 BLSy1 galaxies to whom $\Gamma_{X}^{h}$ had been obtained (e.g. Sect. 4.2). The analysis of these two subsamples along with their joint subsample (i.e., [NLSy1+BLSy1]) resulted in a good positive correlation between $\Gamma_{X}^{h}$ and $\log \left(\mathrm{R}_{\mathrm{E} d d}\right)$ for 53 NLSy1, 46 BLSy1 and 99 [NLSy1+BLSy1] galaxies with $\rho=0.42$, 0.43 , and 0.56 , respectively. This can be seen in the top panels of Fig. 8, and also from the middle part of Table 5. Mild anticorrelations are found between $\Gamma_{X}^{h}$ and $\log (\operatorname{FWHM}(H \beta))$ for the subsamples of NLSy1, BLSy1 and [NLSy1+BLSy1] galaxies with $\rho=-0.37,-0.36$, and -0.48 , respectively. However, no significant correlations are found for $\Gamma_{X}^{h}-\log \left(L_{\mathrm{bol}}\right)$ and $\Gamma_{X}^{h}-\log \left(M_{\mathrm{B} H}\right)$ in 
these subsamples (e.g., see Table 5 and Fig. 8). The $\chi^{2}$ minimization using the functional form of $y=m x+c$ (see above) with $y=\Gamma_{X}^{h}$ and $\mathrm{X}$ either $\log \left(R_{\mathrm{E} d d}\right)$ or $\log (F W H M(H \beta))$, yielded for the subsample of NLSy1 galaxies as

$$
\begin{array}{r}
\Gamma_{X}^{h}=(0.29 \pm 0.06) \log \left(R_{\mathrm{E} d d}\right)+(2.33 \pm 0.03) \\
\Gamma_{X}^{h}=(-0.70 \pm 0.13) \log (F W H M(H \beta))+(4.35 \pm 0.41)
\end{array}
$$

, for the subsample of BLSy1 galaxies as

$$
\begin{array}{r}
\Gamma_{X}^{h}=(0.17 \pm 0.09) \log \left(R_{\mathrm{E} d d}\right)+(2.03 \pm 0.14) \\
\Gamma_{X}^{h}=(-0.26 \pm 0.20) \log (F W H M(H \beta))+(2.71 \pm 0.73)
\end{array}
$$

and for the joint subsample of [NLSy1+BLSy1] galaxies as

$$
\begin{array}{r}
\Gamma_{X}^{h}=(0.35 \pm 0.03) \log \left(R_{\mathrm{E} d d}\right)+(2.34 \pm 0.03) \\
\Gamma_{X}^{h}=(-0.68 \pm 0.06) \log (F W H M(H \beta))+(4.27 \pm 0.19)
\end{array}
$$

as shown by solid red lines in the plots of Fig. 8 .

Additionally, the soft $(0.1-2.0 \mathrm{keV}) \mathrm{X}$-ray photon indices of most of the NLSy1s are affected by soft excess and many of them also by absorption features due to "warm absorbers" (Vaughan et al. 1999). Therefore, a detailed X-ray spectral analysis in the $0.3-10 \mathrm{keV}$ energy band is also carried out to confirm the aforementioned correlations found for the samples of NLSy1 and BLSy1 galaxies between $\Gamma_{X}^{s}$ and $\log \left(\mathrm{R}_{\mathrm{E} d d}\right)$. For this, we analyzed 139 NLSy1 and 97 BLSy1 galaxies in 0.3-10 keV energy band (e.g. Sect. 2) of XMM-Newton Telescope. The analysis of these two subsamples along with their joint subsample (i.e., [NLSy1+BLSy1]) resulted in a good positive correlation between $\Gamma_{X}^{T}$ and $\log \left(\mathrm{R}_{\mathrm{E} d d}\right)$ for NLSy1, BLSy1 and [NLSy1+BLSy1] galaxies subsamples with $\rho$ $=0.42,0.47$, and 0.56 , respectively. This can be seen in the top panels of Fig. 9, and also from the bottom part of Table 5. Mild anticorrelations are found between $\Gamma_{X}^{T}$ and $\log (\operatorname{FWHM}(H \beta))$ for the subsamples of NLSy1s, BLSy1s with $\rho=-0.30,-0.41$, respectively. This is found to be stronger with $\rho$ of -0.52 when both subsamples are combined together. However, no significant correlations are found for $\Gamma_{X}^{T}-\log \left(L_{\mathrm{bol}}\right)$ in these subsamples except a mild anticorrelations found for $\Gamma_{X}^{T}-\log \left(M_{\mathrm{B} H}\right)$ with $\rho$ of -0.30 and -0.41 , in the case of 97 BLSy1 and 236 [NLSy1+BLSy1] galaxies respectively, (e.g., see Table 5 and Fig. 9). The $\chi^{2}$-minimization using the functional form of $y=m x+c$ (see above) with $y=\Gamma_{X}^{T}$ and $\mathrm{X}$ either $\log \left(R_{\mathrm{E} d d}\right)$ or $\log (F W H M(H \beta))$, yielded for the subsample of NLSy1 galaxies as

$$
\begin{array}{r}
\Gamma_{X}^{T}=(0.51 \pm 0.03) \log \left(R_{\mathrm{E} d d}\right)+(2.91 \pm 0.03) \\
\Gamma_{X}^{T}=(-1.32 \pm 0.08) \log (F W H M(H \beta))+(6.73 \pm 0.25)
\end{array}
$$

, for the subsample of BLSy1 galaxies as

$$
\begin{array}{r}
\Gamma_{X}^{T}=(0.34 \pm 0.02) \log \left(R_{\mathrm{E} d d}\right)+(2.56 \pm 0.04) \\
\Gamma_{X}^{T}=(-0.76 \pm 0.05) \log (F W H M(H \beta))+(4.87 \pm 0.17)
\end{array}
$$

and for the joint subsample of [NLSy1+BLSy1] galaxies as

$$
\begin{array}{r}
\Gamma_{X}^{T}=(0.50 \pm 0.01) \log \left(R_{\mathrm{E} d d}\right)+(2.86 \pm 0.02) \\
\Gamma_{X}^{T}=(-0.91 \pm 0.02) \log (F W H M(H \beta))+(5.42 \pm 0.07)
\end{array}
$$

as shown by solid red lines in the plots of Fig. 9 .

Additionally, in view of the steeper $\Gamma_{X}^{s}$ for the sample of NLSy1s and recalling that NLSy1 galaxies do have smaller FWHM of the emission lines as compared to BLSy1 galaxies. It will be worth to explore the possible correlation between FWHM of emission lines and $\mathrm{X}$-ray spectral indices as well. Therefore in the bottom panels of Figs. 7, $8 \&$ 9, we have plotted $\Gamma_{X}^{s}, \Gamma_{X}^{h} \&$ $\Gamma_{X}^{T}$ versus $\log (\operatorname{FWHM}(\mathrm{H} \beta))$ in the soft, hard and total 0.3-10 keV energy bands, respectively. As can be seen from these figures (bottom right panel) that the anticorrelation in $\Gamma_{X}^{s}, \Gamma_{X}^{h} \& \Gamma_{X}^{T}$ versus $\log (\operatorname{FWHM}(\mathrm{H} \beta))$ plots, based on the joint sample of NLSy1 and BLSy1 galaxies is significant with $\rho=-0.55,-0.48$ and -0.52 , with their corresponding $\mathrm{P}_{\text {null }}$ of $2.87 \times 10^{-31}, 3.57 \times 10^{-7}$ and $4.49 \times 10^{-18}$, respectively (see, Table 5 ).

\subsection{Comparison of the $\Gamma_{X}^{\text {hot }}$ correlations with $A G N$ parameters among the subsamples NLSy1 and BLSy1 galaxies}

As detailed in Sect. 4.4 that we could achieve spectral fit in $0.3-10 \mathrm{keV}$ energy band for 34 NLSy1 and 30 BLSy1 galaxies, based on the 'AGNSED' model. This allowed us to estimate the hot spectral indices $\left(\Gamma_{X}^{\text {hot }}\right)$ for these sources along with its correlations with $\mathrm{R}_{\mathrm{E} d d}^{\mathrm{SED}}, \mathrm{L}_{\mathrm{b} o l}$, $\mathrm{M}_{\mathrm{B} H}$ and $\mathrm{FWHM}(\mathrm{H} \beta)$. The plots of these correlations analysis are shown in Fig. 10 and the results are listed in the upper part of Table 6. From this table and the figure, it is clear that the correlations of $\Gamma_{X}^{\text {hot }}$ with other parameters of nuclear activities are almost similar to that of correlations obtained in the $0.1-2.0 \mathrm{keV}$ energy band for the NLSy1 (221 sources), BLSy1 (154 sources) and [NLSy1+BLSy1] (375 sources) galaxies.

\section{DISCUSSION AND CONCLUSIONS}

In order to probe the X-ray emission mechanisms in NLSy1 and BLSy1 galaxies, a correlation study among $\mathrm{X}$-ray spectral indices and parameters of nuclear activity such as $\mathrm{R}_{\mathrm{E} d d}, \mathrm{~L}_{\mathrm{bol}}, \mathrm{M}_{\mathrm{B} H}$, and $\mathrm{FWHM}(\mathrm{H} \beta)$ would be very important. For instance, Brandt et al. (1997) analyzed an Advanced Satellite for Cosmology and Astrophysics (ASCA) sample of 15 NLSy1 and 19 BLSy1 galaxies, for the comparison of their hard X-ray $(2-10 \mathrm{keV})$ photon indices and found that NLSy1s have steeper intrinsic hard X-ray photon indices than the BLSy1s. Here, we have extended similar work by studying the soft (0.1-2.0 $\mathrm{keV})$, hard $(2-10 \mathrm{keV})$ as well as total $(0.3-10 \mathrm{keV})$ photon indices (i.e., $\Gamma_{X}^{s}, \Gamma_{X}^{h} \& \Gamma_{X}^{T}$ ) of NLSy1 and BLSy1 galaxies. For this, we have constructed their samples based 

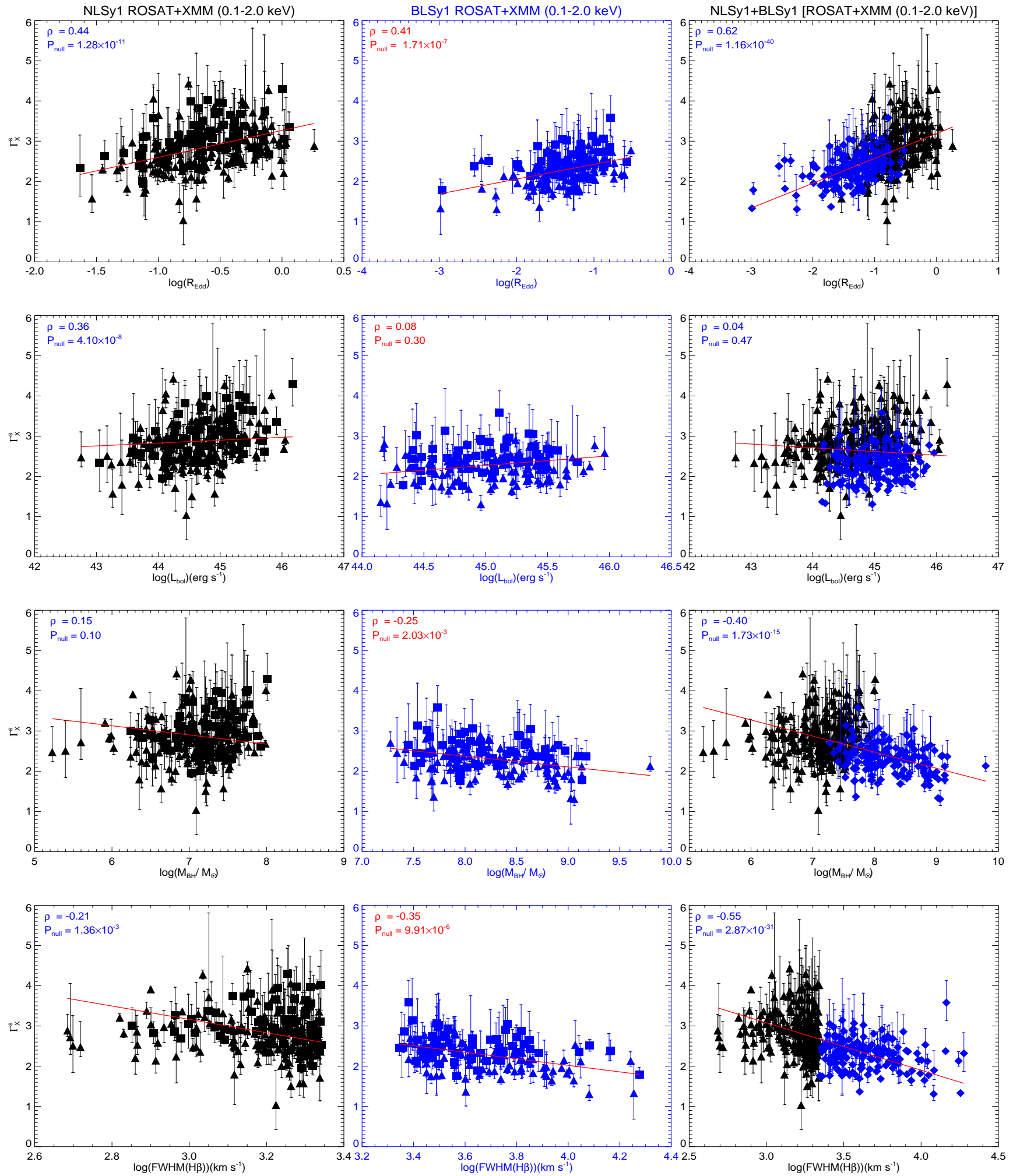

Fig. 7.- Correlations of the 221 NLSy1s (left) and 154 BLSy1s (middle) either from the ROSAT (filled squares) or from the XMM-Newton (filled triangles) for the soft (0.1-2.0 keV) X-ray photon indices $\left(\Gamma_{X}^{s}\right)$ versus Eddington ratios, bolometric luminosities, black hole masses and FWHM of $\mathrm{H} \beta$ lines, respectively from top to bottom panels, along with their error-weighted linear fit (red, solid line). The last column presents the correlations among the same, but for the joint sample of 221 NLSy1s (black, filled triangles) and 154 BLSy1s (blue, filled diamonds). The plots also give the Spearman's correlation coefficient $(\rho)$ and the probability of null correlation $\left(\mathrm{P}_{\text {null }}\right)$ values (left corner of each panel).

on the recent large catalog of 11,101 NLSy1s and their redshift matched sample of BLSy1s using their X-rays data from the ROSAT and XMM-Newton (e.g. Sect. 2).
Our sample consist of 221 NLSy1, 154 BLSy1 galaxies in soft (0.1-2.0 keV), 53 NLSy1, 46 BLSy1 galaxies in the hard (2-10 keV), and 139 NLSy1, 97 BLSy1 galaxies in 

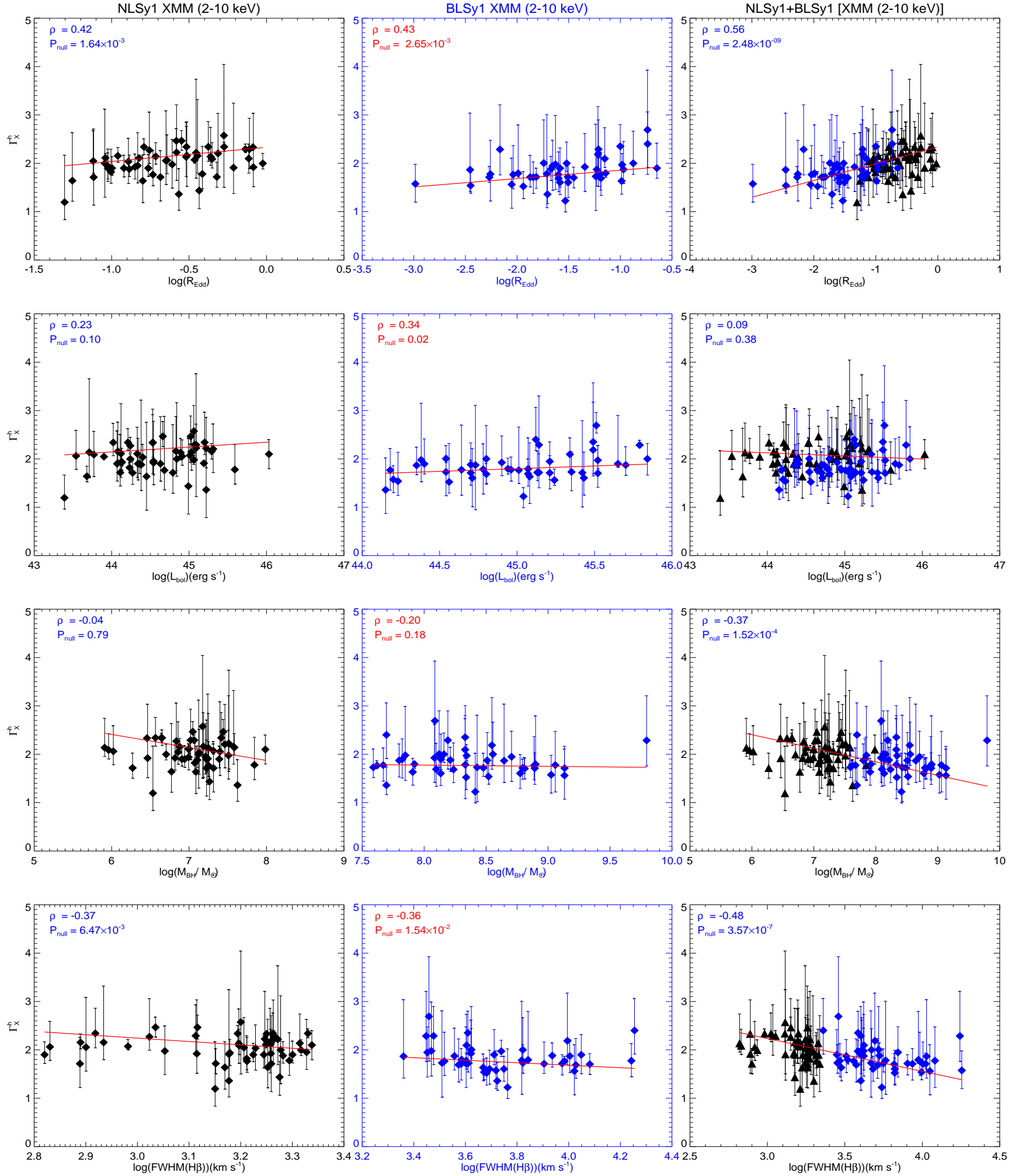

FIG. 8. - Same as Fig. 7, but using the 53 NLSy1 and 46 BLSy1 galaxies for the $X M M-N e w t o n$ hard (2-10 keV) X-ray photon indices ( $\Gamma_{X}^{h}$ ).

the total $(0.3-10 \mathrm{keV})$ energy bands (e.g. Sect. 2). A homogeneous analysis is carried out for the estimations of $\Gamma_{X}^{s}, \Gamma_{X}^{h}, \Gamma_{X}^{T}$ and other parameters of nuclear activities such as $\mathrm{R}_{\mathrm{E} d d}, \mathrm{~L}_{\mathrm{b} o l}, \mathrm{M}_{\mathrm{B} H}$, and $\operatorname{FWHM}(\mathrm{H} \beta)$ of the NLSy1 and BLSy1 galaxies. This homogeneous analysis is carried out to perform a comparative study between these two subclasses of AGN along with a comparison of them with other classes of luminous AGNs in soft, hard, and total X-ray energy bands.

The advantages of our analysis are that we have used an enlarged sample of NLSy1s (e.g., see Table 1). For comparison, we have used a control sample of BLSy1s, 

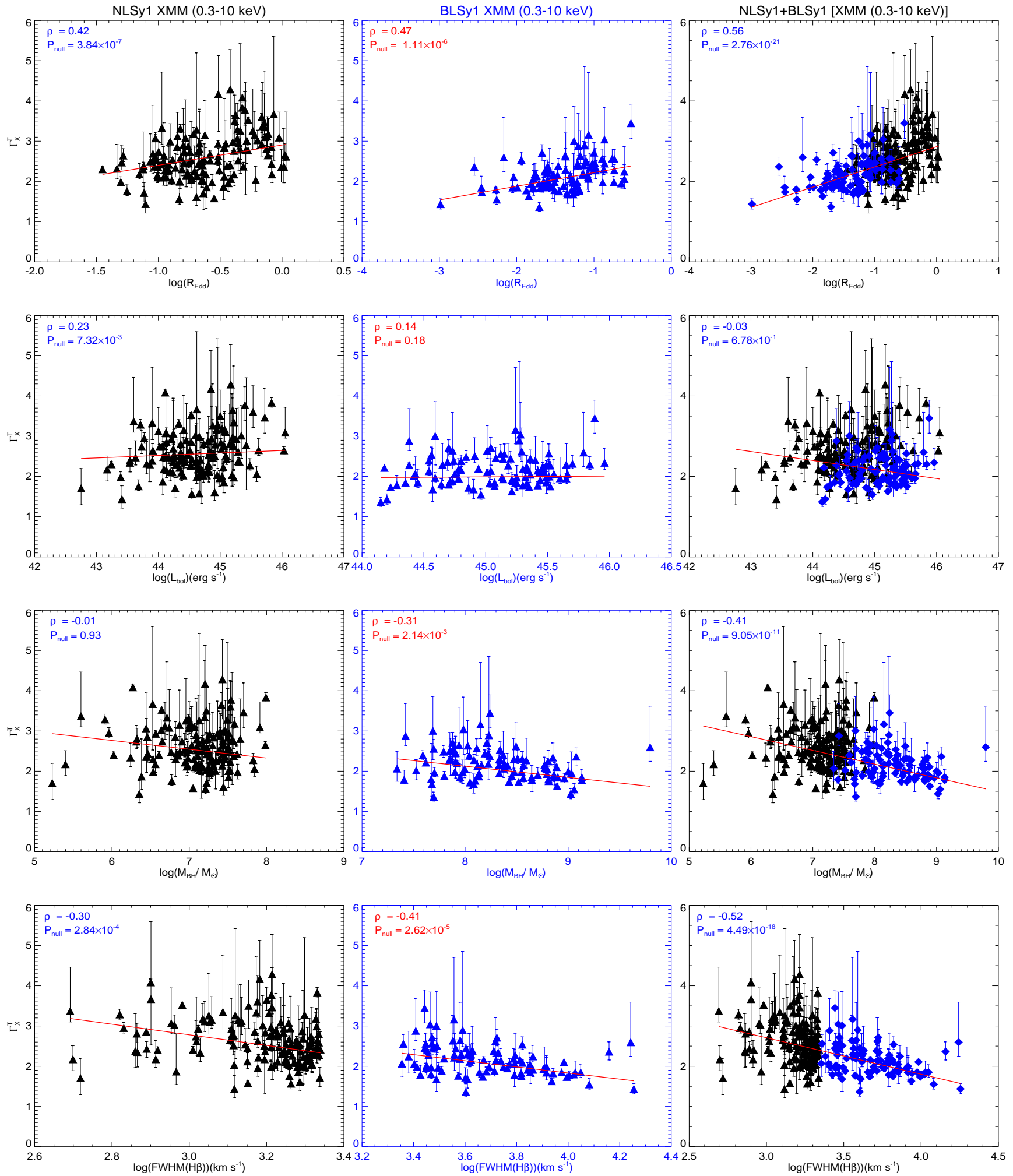

FIG. 9.- Same as Fig. 7, but using the 139 NLSy1 and 97 BLSy1 galaxies for the XMM-Newton total (0.3-10 keV) X-ray photon indices ( $\left.\Gamma_{X}^{T}\right)$.

matching (moderately) in the redshift plane (e.g., see Fig. 1 and Sect. 2). Furthermore, in our analysis, to compute the soft, hard and total energy X-ray photon indices, we have used similar models (mostly) in the soft, hard and total energy X-ray bands. This extra caution is taken care in our method so as to avoid the variations in the estimated $\Gamma_{X}^{s}, \Gamma_{X}^{h}$ and $\Gamma_{X}^{T}$ due to the use of different spectral fitting models, as has been the case in many previous studies as mentioned in Sect. 1.

The main results of our systematic homogeneous analysis presented here are as follows. Firstly, we found a clear significant difference among the $\Gamma_{X}^{s}$ distribution 

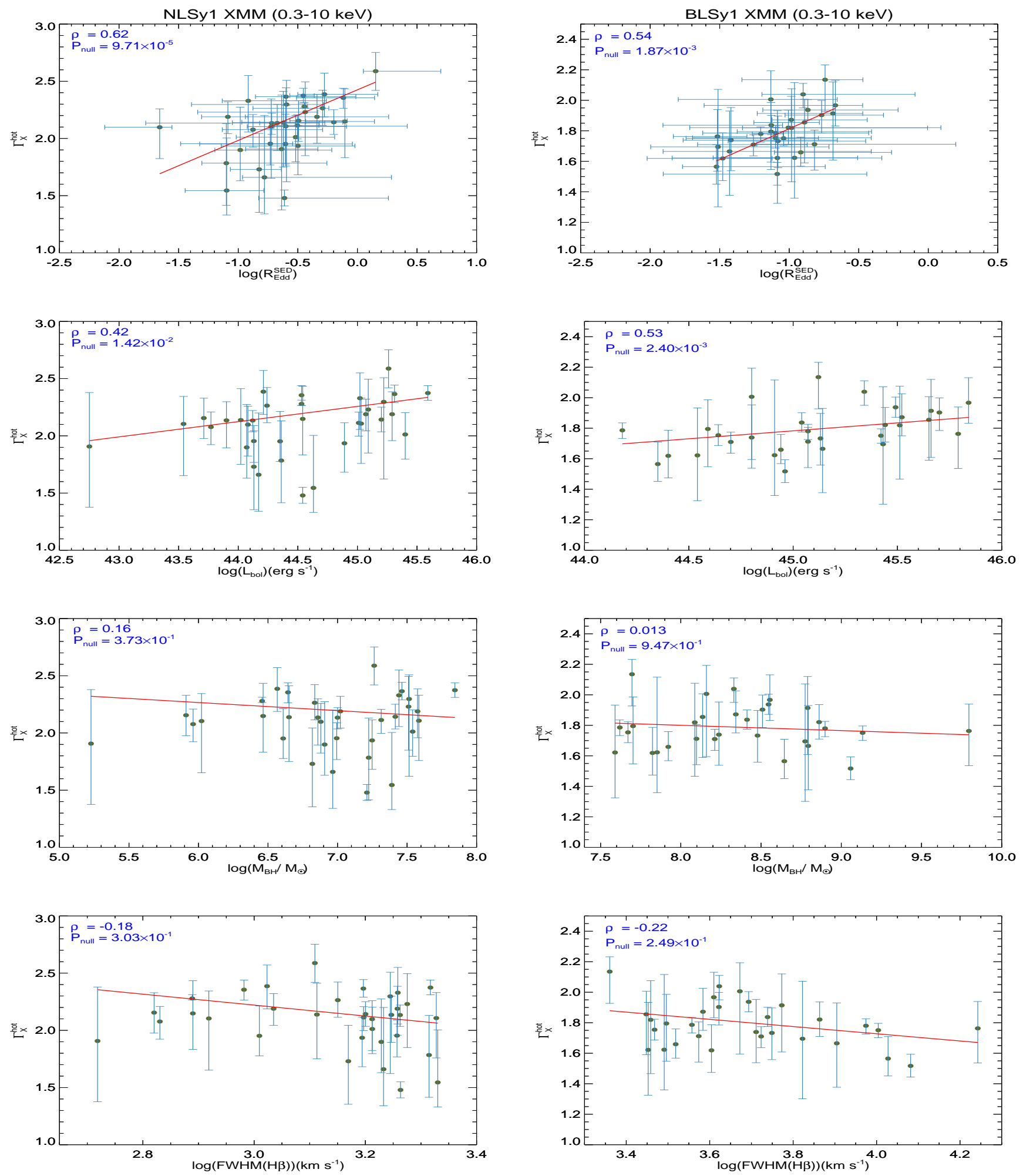

Fig. 10. - Same as Fig. 7, but using the spectral fit of 34 NLSy1 and 30 BLSy1 galaxies based on the AGNSED model for the X-ray hot photon indices $\left(\Gamma_{X}^{\text {hot }}\right)$ in the $0.3-10 \mathrm{keV}$ energy band of the XMM-Newton data.

of NLSy1 and BLSy1 galaxies (being steeper for the NLSy1 class, e.g., see Fig. 3) with median values of 2.81 and 2.30 for the samples of the NLSy1 and BLSy1 galaxies, respectively, having $\mathrm{P}_{\text {null }}$ of $4.02 \times 10^{-19}$ based on the K-S test. One reason for this observed differ- ence among the $\Gamma_{X}^{s}$ distribution of NLSy1 and BLSy1 galaxies could be more soft X-ray excess in NLSy1s. To lift this degeneracy, we have compared $\Gamma_{X}^{h}$, which are thought to be free from the soft X-ray excess (e.g., see Boller et al. 1996; Brandt et al. 1997; Vaughan et al. 

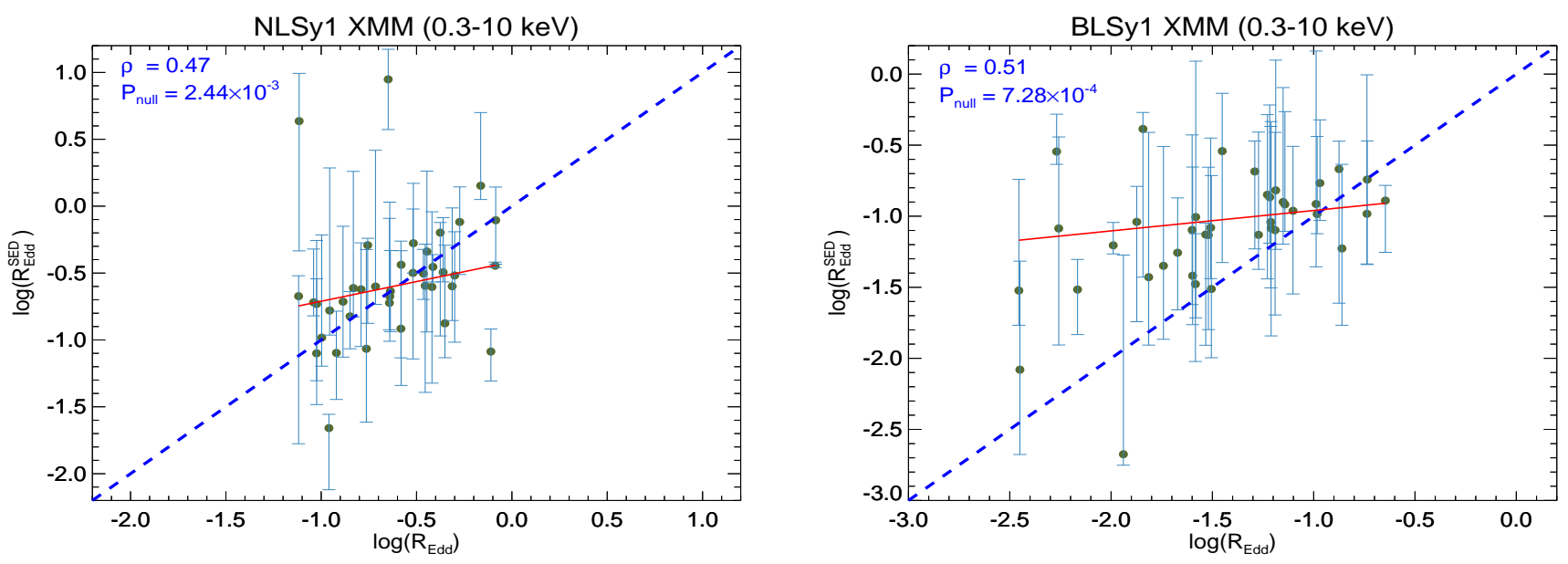

FIG. 11.- Correlations of 34 NLSy1s (left) and 30 BLSy1s (right) for the X-ray Eddington ratio $\left(\log \left(\mathrm{R}_{\mathrm{E} d d}^{\mathrm{SED}}\right)\right.$ ) and optical Eddington ratio $\left(\log \left(\mathrm{R}_{\mathrm{E} d d}\right)\right)$ obtained from X-ray fitting in $0.3-10 \mathrm{keV}$ energy band using the AGNSED model and optical scaling relationship (see, Sect. 4.1), respectively. The dotted blue line is plotted in each panel following the equation $y=x$.

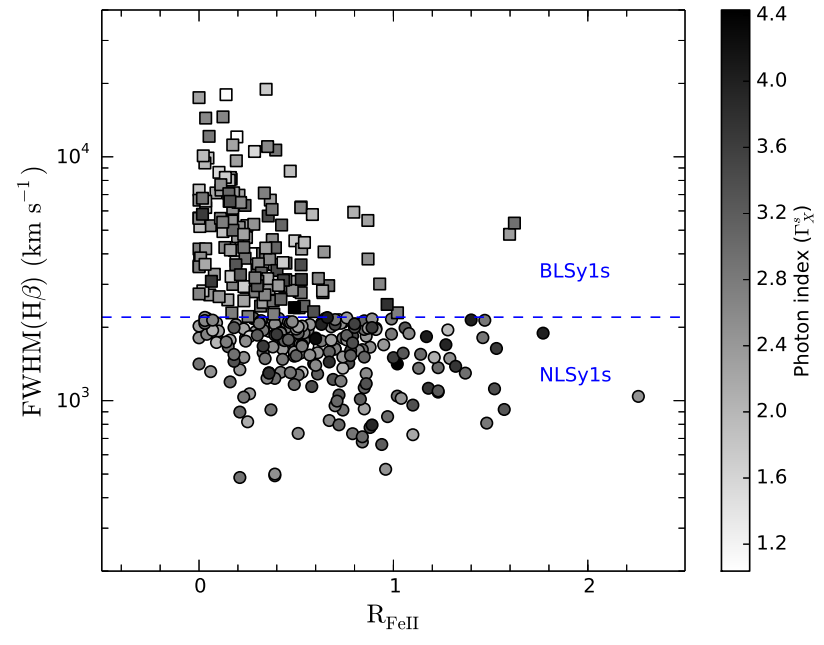

Fig. 12.- A plot of Eigenvector $1 \mathrm{EV} 1\left(\mathrm{R}_{\mathrm{FeII}}\right.$ vs $\left.\mathrm{FWHM}(\mathrm{H}(\beta))\right)$ with space gray codded by the soft $(0.1-2.0 \mathrm{keV}) \mathrm{X}$-ray photon indices $\left(\Gamma_{X}^{s}\right.$ ) for the 221 NLSy1 (gray filled circle) and 154 BLSy1 (gray filled square) galaxies.

1999; Boller et al. 2002; Czerny et al. 2003; Vignali et al. 2004 ) between the subsamples of 53 NLSy1 and 46 BLSy1 galaxies based on their 2-10 keV XMM-Newton observations. In this comparison also we find a difference in $\Gamma_{X}^{h}$ with median values of 2.06 and 1.78 , having $\mathrm{P}_{\text {null }}$ of $1.00 \times 10^{-3}$ for the subsamples of NLSy1 and BLSy1 galaxies, respectively (e.g., see Fig. 4). This confirms that the above result of the difference in $\Gamma_{X}^{s}$ distribution is unlikely to be solely due to the soft X-ray excess and rather seems to be intrinsic in their nature. Furthermore, we noticed that the difference in the median photon indices of hard energy band subsamples of NLSy1 \& BLSy1 galaxies is statistically weaker than the soft energy band samples of NLSy1 and BLSy1 galaxies. This may be due to comparatively about 4 times smaller hard band subsamples of NLSy1 \& BLSy1 galaxies. To lift this degeneracy, we have compared the $\Gamma_{X}^{T}$ between the subsamples of 139 NLSy1 and 97 BLSy1 galaxies. We again find a significant difference with median values of 2.53 and 2.13 , respectively, having $P_{\text {null }}$ of $4.50 \times 10^{-9}$ which is consistent with the result based on soft X-ray analysis of these NLSy1 and BLSy1 galaxies.

Secondly, to ascertain whether there is a bimodality or continuity in X-ray spectral indices (hereafter $\Gamma_{X}$ will be referring to $\Gamma_{X}^{s}, \Gamma_{X}^{h}$ and $\Gamma_{X}^{T}$ ) among NLSy1 and BLSy1 galaxies. A detailed correlation analysis of $\Gamma_{X}$ with other physical parameters of AGNs such as $\mathrm{R}_{\mathrm{E} d d}, \mathrm{~L}_{\mathrm{bol}}, \mathrm{M}_{\mathrm{B} H}$, and $\operatorname{FWHM}(\mathrm{H} \beta)$ is carried out. This correlation analysis results in the strongest $\Gamma_{X}-\log \left(R_{\mathrm{E} d d}\right)$ correlation for the samples of NLSy1 and BLSy1 galaxies (e.g. Sect. 5.2 and Table 5), implying that $\mathrm{R}_{\mathrm{E} d d}$ may be the dominant parameter related to $\Gamma_{X}$. Additionally, the joint analysis of [NLSy1+BLSy1] shows that the variation seems to be continuous rather than a clear significant bimodality in its distribution (e.g., see last column of Figs. 7, 8 \& 9). This is also evident in their joint histogram plots of $\Gamma_{X}$ which do not show two well-separated significant peaks (e.g., see histogram of joint (NLSy1+BLSy1) distribution in Figs. 3, 4 \& 5). Furthermore, the similarity of the trends and value of Spearman's correlations for $\Gamma_{X}-\log \left(R_{\mathrm{E} d d}\right)$ correlation found for NLSy1 and BLSy1 galaxies in the soft, hard and total X-ray energy bands (e.g., see Table 5) also suggest that their emission mechanism may be similar. However, the slopes of the linear fit of $\Gamma_{X}$ and $\log \left(\mathrm{R}_{\mathrm{E} d d}\right)$ correlations do differ significantly among the samples of NLSy1 and BLSy1 galaxies in soft, hard and total X-ray energy bands (e.g., see Table 5) which could probably be due to the difference in their accretion rates, being higher for the former.

We explored this possibility by comparing the distribution of $\mathrm{R}_{\mathrm{E} d d}$ of NLSy1 and BLSy1 galaxies. This has resulted in the median values of $\mathrm{R}_{\mathrm{E} d d}, 0.23$ and 0.05 for 221 NLSy1 and 154 BLSy1 galaxies, respectively, in the soft X-ray. The $\mathrm{R}_{\mathrm{E} d d}$ distribution differs significantly for the above samples of NLSy1 and BLSy1 galaxies with KS test based $P_{\text {null }}=2.66 \times 10^{-35}$. The above result also holds when we compare in $0.3-10 \mathrm{keV}$ energy band. The distributions of $\mathrm{R}_{\mathrm{E} d d}$ of 139 NLSy1 and 97 BLSy1 galaxies, having median values of 0.22 and 0.04 respectively, and found that their K-S test based $P_{\text {null }}$ is $3.72 \times 10^{-26}$. 
Similarly, using the analysis of 53 NLSy 1 and 46 BLSy 1 galaxies in hard energy band, the median values of $\mathrm{R}_{\mathrm{E} d d}$ are found to be 0.25 and 0.03 , respectively, resulting in K-S test based $P_{\text {null }}$ of $1.72 \times 10^{-14}$. In view of the above, negligible dependence of the $P_{\text {null }}$ values on the energy bands used in the analysis, allows to concluded that the $\mathrm{R}_{\mathrm{E} d d}$ of NLSy1 and BLSy1 galaxies seem to be intrinsically significantly different, being it higher for the former.

To reconcile this discrepancy one possibility is that the higher $\mathrm{R}_{\mathrm{E} d d}$ in NLSy1s (compared to BLSy1s) can be due to the fact that the inclination angle of the NLSy1 is lower than that of the BLSy1. As a result, the observed FWHM (in $\mathrm{km} \mathrm{s}^{-1}$ ) of $\mathrm{H} \beta$ line $(\mathrm{FWHM} \times \sin (\theta)$ ) of the BLR would have been underestimated more in the case of NLSy1 (due to smaller inclination) compared to the BLSy1 (e.g., see Baldi et al. 2016; Liu et al. 2016; Rakshit et al. 2017). This will directly impact the underestimation of their $\mathrm{M}_{\mathrm{B} H}$ (being proportional to the observed FWHM in the common L- $\mathrm{R}_{B L R}$ scaling relationship) and hence the over-estimation of the $R_{\mathrm{E} d d}$ value (being inversely proportional to $\mathrm{M}_{\mathrm{B} H}$ ). To consider such projection effect of BLR for the NLSy1 and BLSy1 galaxies in our analysis, we corrected the observed FWHM (i.e., projected) values as $F W H M / \sin (\theta)$, by using the median viewing angle $(\theta)$ of $13.6^{\circ}$ and $27.7^{\circ}$ as given by Liu et al. (2016) for the NLSy1s and BLSy1s, respectively. This has allowed us to have corrected $\mathrm{M}_{\mathrm{B} H}$ and $\mathrm{R}_{\mathrm{E} d d}$ for our samples of NLSy1 and BLSy1 galaxies. The corrected $\mathrm{R}_{\mathrm{E} d d}$ values still show a difference (though with less statistical significance) among its distribution in 221 NLSy1 and 154 BLSy1 galaxies with K-S test based $P_{\text {null }}=3.45 \times 10^{-2}$. The difference still exists when we even use only the subsamples of 139 NLSy1 and 97 BLSy1 galaxies analyzed in $0.3-10 \mathrm{keV}$ energy range, giving $P_{\text {null }}=2.18 \times 10^{-2}$. Here, a possibility also exists that it may also be due to the imperfection of exact luminosities matching and/or due to our application of average inclination angle value for the entire sample. Nonetheless, given that the mismatch in luminosities in our sample is nominal, and the fact that the $\mathrm{R}_{\mathrm{Edd}}$ difference being very significant, suggest that it is intrinsically higher in NLSy1 compare to BLSy1. This could lead to the above measured differences in $\Gamma_{X}^{s}, \Gamma_{X}^{h}$, $\Gamma_{X}^{T}$ distributions and the slopes of the linear fit of the $\Gamma_{X}$ and $\log \left(R_{\mathrm{E} d d}\right)$ correlations.

Another possibility for the above difference in the $\mathrm{R}_{\mathrm{E} d d}$ can be due to under/over estimations of bolometric luminosity, which was estimated using the scaling relationship of $\mathrm{L}_{\mathrm{bol}}$ and optical luminosity at $5100 \AA$ (e.g. Sect. 4.2). To quantify its effect, we have also estimated the $\mathrm{R}_{\mathrm{E} d d}$ values independently by fitting the AGNSED model over the $0.3-10 \mathrm{keV}$ band (i.e., $\mathrm{R}_{\mathrm{E} d d}^{\mathrm{SED}}$ ) for the 34 NLSy1 and 30 BLSy1 galaxies subsamples (e.g. Sect. 4.4). The resulting distribution of $\mathrm{R}_{\mathrm{E} d d}^{\mathrm{SED}}$ (e.g., see Fig. 6) have also shown a significant difference (with $\mathrm{P}_{\text {null }}=2.01 \times 10^{-7}$ ) in the subsamples of these 34 NLSy1 and 30 BLSy1 galaxies. This is consistent with the conclusion drawn using $\mathrm{R}_{\mathrm{Edd}}$ distribution, based on $\mathrm{L}_{\mathrm{bol}}$ estimated using the optical spectra (e.g., see Figs. 3, 4, 5). This is not surprising because there is a significant correlation between $\mathrm{R}_{\mathrm{E} d d}$ (i.e., using optical) and $\mathrm{R}_{\mathrm{E} d d}^{\mathrm{S} E D}$ (i.e., using X-ray) for the NLSy1 and BLSy1 galaxies as shown in Fig. 11 and tabulated in the last row of Table 6. Furthermore, the histograms of spectral indices estimated using $A G N S E D$ model $\left(\Gamma_{X}^{S E D}\right.$, also presented as $\Gamma_{X}^{\text {hot }}$ in Fig. 10) also show a significant difference among NLSy1 and BLSy1 galaxies with $\mathrm{P}_{\text {null }}$ of $8.87 \times 10^{-6}$ (e.g., see Fig. 6). In addition to this, a correlation between $\Gamma_{X}^{\text {hot }}$ and $\log \left(R_{\mathrm{E} d d}^{\mathrm{S} E D}\right)$ is also found in the above subsamples of NLSy1 and BLSy1 galaxies (e.g., see Fig. 10) which is similar to the correlations found in the soft, hard and total energy bands between $\Gamma_{X}$ and $\mathrm{R}_{\mathrm{E} d d}$.

Our above investigations suggest that the $\mathrm{R}_{\mathrm{E} d d}$ of NLSy1 is unambiguously higher than that of BLSy1 galaxy. This intrinsic difference can explain our observed significant difference of spectral indices among NLSy1 and BLSy1 galaxies as follow.

The higher value of $\mathrm{R}_{\mathrm{E} d d}$ can lead to an increase in the disk temperature, hence producing more X-ray radiations, and at the same time, it can also increase the Compton cooling of corona (Haardt \& Maraschi 1991, 1993; Zdziarski et al. 2000; Kawaguchi et al. 2001), which leads to steepening of the X-ray power-law more in NLSy1 than BLSy1, and hence will lead to the observed difference we noticed in our spectral indices in the soft, hard and total $0.3-10 \mathrm{keV}$ energy bands (e.g. Sect. 5.1). Moreover, this also could be the reason for the observed higher slope of $\Gamma_{X}$ and $\log \left(R_{\mathrm{E} d d}\right)$ linear fit for NLSy1 compared to the BLSy1 galaxies (e.g., see Table 5), as such stronger dependence in case of NLSy1 can be reconciled due to their higher $\mathrm{R}_{\mathrm{E} d d}$ value.

As pointed out in Sect. 1, such positive $\Gamma_{X}^{h}-\log \left(R_{\mathrm{E} d d}\right)$ correlation (e.g., see Table 5) has also been found for the luminous AGNs by Risaliti et al. (2009). It may be noted that they found a $\rho$ of 0.32 based on their sample of 343 AGNs. However, this correlation becomes stronger with the value of $\rho=0.56$ when considering only their subset of 82 objects whose black hole masses were estimated using their $\mathrm{H} \beta$ lines. This is almost similar to the Spearman's correlation coefficients found for the $\Gamma_{X}^{s}-\log \left(R_{\mathrm{E} d d}\right), \Gamma_{X}^{h}-\log \left(R_{\mathrm{E} d d}\right)$ and $\Gamma_{X}^{T}-\log \left(R_{\mathrm{E} d d}\right)$ correlations in our samples of the NLSy1 and BLSy1 galaxies (e.g., see Table 5). On the other hand, the striking contrast to this positive correlation as compared to the corresponding negative correlation found for the case of LLAGNs by Gu \& Cao (2009) suggests that emission mechanism in the NLSy1 and BLSy1 galaxies is different as compared to the LLAGNs but likely to be similar to the luminous AGNs.

Finally, we have also explored the correlation of X-ray spectral slopes (in soft energy band) with that of the optical plane of Eigenvector 1 (EV1) which is mainly defined by $\operatorname{FWHM}(\mathrm{H} \beta)$ and the flux ratio of $\mathrm{Fe}$ II to $\mathrm{H} \beta$, $\mathrm{R}_{\mathrm{FeII}}$ (Sulentic et al. 2000). The $\operatorname{FWHM}(\mathrm{H} \beta)$ is known to be affected by the inclination angle while the $\mathrm{R}_{\mathrm{FeII}}$ is driven by the Eddington ratio (Shen \& Ho 2014). The $\mathrm{R}_{\mathrm{FeII}}$ values of NLSy1 galaxies are taken from the parent catalog of NLSy1 galaxies given by Rakshit et al. (2017) and for the BLSy1 galaxies, it is estimated following the similar procedure as used in Rakshit et al. (2017) for the NLSy1 galaxies. In Fig. 12, we have plotted these quantities color-coded by the $\Gamma_{X}^{s}$. We find strong Fe II emitters to have steeper photon indices compared to the week Fe II emitters. The Spearman's rank correlation coefficients between $\mathrm{R}_{\mathrm{FeII}}$ and $\Gamma_{X}^{s}$ are found to be 0.31 and 0.24 for the samples of NLSy1 and BLSy1 galaxies 
while 0.46 when both NLSy1 and BLSy1 samples are combined together. This positive correlation between $\Gamma_{X}^{s}$ and $\mathrm{R}_{\mathrm{F} e I I}$ reflects the strong correlation found between photon indices and Eddington ratios. Moreover, Fig. 12 also corroborate the strong anticorrelation between photon indices and $\mathrm{FWHM}(\mathrm{H} \beta)$ found in the joint analysis of NLSy1 and BLSy1 samples (e.g. Sect. 5.2 and Figs. $7,8,9)$. In view of such a strong anti-correlation, we may recall that $\Gamma_{X}$ being related to the X-ray emitting regions is generally much closer to the central engine of AGN as compared to BLR clouds whose broadening is measured as the FWHM of $\mathrm{H} \beta$ lines. As a result, such strong anticorrelation between these two seemingly disconnected regions are worth noting while constructing any models of the AGNs emission mechanisms along with the observed strong correlation of the $\Gamma_{X}-\log \left(R_{\mathrm{E} d d}\right)$ found both for NLSy1 and BLSy1 galaxies.

\section{SUMMARY}

In this work, we have quantitatively compared NLSy1 and BLSy1 galaxies for their X-ray and optical properties along with different correlations among X-ray and optical parameters, such as $\Gamma_{X}-\log \left(R_{\mathrm{E} d d}\right), \Gamma_{X}-\log \left(L_{\mathrm{bol}}\right)$, $\Gamma_{X}-\log \left(M_{\mathrm{B} H}\right)$ and $\Gamma_{X}-\log (F W H M(H \beta))$ with $\Gamma_{X}$ representing X-ray spectral slope either in soft (i.e. $\Gamma_{X}^{s}$ in $0.1-2.0 \mathrm{keV}$ ), in hard (i.e. $\Gamma_{X}^{h}$ in $2-10 \mathrm{keV}$ ) and in total (0.3-10 keV) energy bands (i.e. $\Gamma_{X}^{T}$ ). For these, we used the samples of 221 NLSy1 and 154 BLSy1 galaxies in the soft X-ray energy (0.1-2.0 keV) band, while its subsamples of 53 NLSy1, 46 BLSy1 and 139 NLSy1, 97 BLSy1 galaxies are used also in the hard X-ray and total energy bands, respectively. The summary of our main results is as follows.

(i) We found the existence of difference in $\Gamma_{X}^{s}$ distribution among the NLSy1 and BLSy1 galaxies, being steeper for the former in the soft X-ray energy band. Furthermore, the difference is also found when spectral indices of these two subsets are compared in hard and total energy bands (i.e., $\Gamma_{X}^{h}$ and $\Gamma_{X}^{T}$ ). In view of the fact that the hard energy band is generally less prone to the impact of soft X-ray excess, it suggests that soft X-ray excess is not the main cause of the difference seen here among the $\Gamma_{X}$ found for the NLSy1 and BLSy1 galaxies.

(ii) We found a clear significant difference in $R_{\mathrm{E} d d}$ among NLSy1 and BLSy1 galaxies, being larger for the former with $\mathrm{P}_{\text {null }}$ of $2.66 \times 10^{-35}$. This difference exists even after incorporating any inclination angle difference among these two subclasses, though with less significance. Furthermore, this discrepancy (based on $\mathrm{R}_{\mathrm{E} d d}$ from the optical data) is also reconfirmed even when we estimated $\mathrm{R}_{\mathrm{E} d d}$ independently based on SED fitting (AGNSED model) in the X-ray $0.3-10 \mathrm{keV}$ band (e.g. Sect. 4.4). This suggests that $\mathrm{R}_{\mathrm{E} d d}$ of the NLSy1 is intrinsically higher than the BLSy1 galaxy which can be the main reason for the observed significant difference in $\Gamma_{X}$ among NLSy1 and BLSy1 galaxies in soft, hard and total energy bands.

(iii) Our analysis suggests a significant positive correlation between $\Gamma_{X}$ and $\log \left(\mathrm{R}_{\mathrm{E} d d}\right)$ for the samples of NLSy1 and BLSy1 galaxies in soft, hard and total energy bands, with stronger dependence in the case of NLSy1s. Also, these strong correlations between $\Gamma_{X}$ and $\log \left(\mathrm{R}_{\mathrm{E} d d}\right)$ for the NLSy1 and BLSy1 galaxies show that their X-ray slope can be used as an Eddington ratio estimator, which can then also be used to calculate black hole mass for a given bolometric luminosity estimate.

(iv) Overall correlations such as $\Gamma_{X}-\log \left(R_{\mathrm{E} d d}\right)$ have almost similar trends among the NLSy1 and BLSy1 galaxies. These correlations are found also consistent (qualitatively) with the luminous AGNs (at least in hard X-ray), apart from their higher significance compare to NLSy1 and BLSy1 galaxies. This gives support to the theoretical prediction that, the X-ray emissions may also be produced in NLSy1 and BLSy1 galaxies by the disccorona system as proposed for the case of luminous AGNs. This model is also consistent with the steeper $\Gamma_{X}$ we have found in our samples of NLSy1 in comparison to the BLSy1 galaxies.

We thank the anonymous referee for the constructive comments on our manuscript. We gratefully acknowledge Mainpal Ranjan, Jeewan C. Pandey and Xinwu Cao for their very useful discussions.

\section{REFERENCES}

Alam, S., Albareti, F. D., Allende Prieto, C., et al. 2015, ApJS, 219, 12

Arnaud, K. A. 1996, in Astronomical Society of the Pacific Conference Series, Vol. 101, Astronomical Data Analysis Software and Systems V, ed. G. H. Jacoby \& J. Barnes, 17

Baldi, R. D., Capetti, A., Robinson, A., Laor, A., \& Behar, E. 2016, MNRAS, 458, L69

Bennett, C. L., Larson, D., Weiland, J. L., \& Hinshaw, G. 2014 ApJ, 794, 135

Boller, T., Brandt, W. N., \& Fink, H. 1996, A\&A, 305, 53

Boller, T., Freyberg, M. J., Trümper, J., et al. 2016 , A\&A, 588, A103

Boller, T., Fabian, A. C., Sunyaev, R., et al. 2002, MNRAS, 329, L1

Boroson, T. A. 2002, ApJ, 565, 78

Boroson, T. A., \& Green, R. F. 1992, ApJS, 80, 109

Brandt, W. N., Mathur, S., \& Elvis, M. 1997, MNRAS, 285, L25

Collin, S., \& Kawaguchi, T. 2004, A\&A, 426, 797

Czerny, B., Nikołajuk, M., Różańska, A., et al. 2003, A\&A, 412, 317

Dickey, J. M.. \& Lockman, F. J. 1990, ARA\&A, 28, 215

Dorman, B., \& Arnaud, K. A. 2001, in Astronomical Society of the Pacific Conference Series, Vol. 238, Astronomical Data Analysis Software and Systems X, ed. F. R. Harnden, Jr., F. A. Primini, \& H. E. Payne, 415
Esin, A. A., McClintock, J. E., \& Narayan, R. 1997, ApJ, 489, 865 Gayathri, V., Bacon, P., Pai, A., et al. 2019, arXiv e-prints, arXiv:1907.10851

González-Martín, O., Masegosa, J., Márquez, I., Guerrero, M. A., \& Dultzin-Hacyan, D. 2006, A\&A, 460, 45

Grupe, D., Beuermann, K., Mannheim, K., \& Thomas, H.-C. 1999, A\&A, 350, 805

Grupe, D., Beuermann, K., Thomas, H.-C., Mannheim, K., \& Fink, H. H. 1998, A\&A, 330, 25

Gu, M., \& Cao, X. 2009, MNRAS, 399, 349

Haardt, F., \& Maraschi, L. 1991, ApJ, 380, L5

-. 1993, ApJ, 413, 507

Kawaguchi, T., Shimura, T. \& Mineshige, S. 2001, ApJ, 546, 966

Klimek, E. S., Gaskell, C. M., \& Hedrick, C. H. 2004, ApJ, 609, 69

Komossa, S., \& Meerschweinchen, J. 2000, A\&A, 354, 411

Kshama, S. K., Paliya, V. S., \& Stalin, C. S. 2017, MNRAS, 466, 2679

Kubota, A., \& Done, C. 2018, MNRAS, 480, 1247

Laor, A., Fiore, F., Elvis, M., Wilkes, B. J., \& McDowell, J. C. 1997, ApJ, 477, 93

Leighly, K. M. 1999, ApJS, 125, 297

Liu, X., Yang, P., Supriyanto, R., \& Zhang, Z. 2016,

International Journal of Astronomy and Astrophysics, 6, 166

Lu, Y., \& Yu, Q. 1999, ApJ, 526, L5 
Maoz, D. 2007, MNRAS, 377, 1696

McLure, R. J., \& Dunlop, J. S. 2004, MNRAS, 352, 1390

Miller, H. R., Ferrara, E. C., McFarland, J. P., et al. 2000, New A Rev., 44, 539

Ojha, V., Krishna, G., \& Chand, H. 2019, MNRAS, 483, 3036

Osterbrock, D. E., \& Pogge, R. W. 1985, ApJ, 297, 166

Paliya, V. S., Sahayanathan, S., Parker, M. L., et al. 2014, ApJ, 789, 143

Panessa, F., Bassani, L., Cappi, M., et al. 2006, A\&A, 455, 173

Pounds, K. A., Done, C., \& Osborne, J. P. 1995, MNRAS, 277, L5

Pounds, K. A., Nandra, K., Fink, H. H., \& Makino, F. 1994, MNRAS, 267, 193

Rakshit, S., \& Stalin, C. S. 2017, ApJ, 842, 96

Rakshit, S., Stalin, C. S., Chand, H., \& Zhang, X.-G. 2017, ApJS, 229, 39

Risaliti, G., Young, M., \& Elvis, M. 2009, ApJ, 700, L6
Schneider, D. P., Hall, P. B., Richards, G. T., et al. 2005, AJ, 130,367

Shen, Y., \& Ho, L. C. 2014, Nature, 513, 210

Shuder, J. M., \& Osterbrock, D. E. 1981, ApJ, 250, 55

Sulentic, J. W., Zwitter, T., Marziani, P., \& Dultzin-Hacyan, D. 2000, Ap. J, 536, L5

Vaughan, S., Reeves, J., Warwick, R., \& Edelson, R. 1999, MNRAS, 309, 113

Vestergaard, M., \& Peterson, B. M. 2006, ApJ, 641, 689 Vignali, C., Brandt, W. N., Boller, T., Fabian, A. C., \& Vaughan, S. 2004, MNRAS, 347, 854

Wandel, A., Milgrom, M., \& Yahil, A. 1985, ApJ, 292, 206

Wang, T., Brinkmann, W., \& Bergeron, J. 1996, A\&A, 309, 81

Zdziarski, A. A., Poutanen, J., \& Johnson, W. N. 2000, ApJ, 542, 703

Zhou, H., Wang, T., Yuan, W., et al. 2006, ApJS, 166, 128 